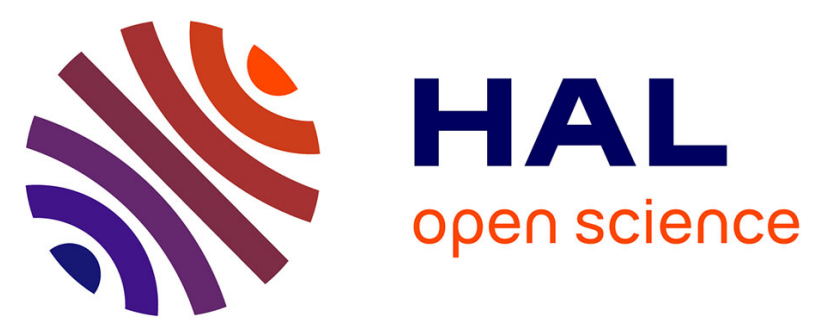

\title{
Intravital dynamic and correlative imaging reveals diffusion-dominated canalicular and flow-augmented ductular bile flux
}

Nachiket Vartak, Georgia Guenther, Florian Joly, Amruta Damle-Vartak, Gudrun Wibbelt, Jörns Fickel, Simone Jörs, Brigitte Begher-Tibbe, Adrian

Friebel, Kasimir Wansing, et al.

\section{To cite this version:}

Nachiket Vartak, Georgia Guenther, Florian Joly, Amruta Damle-Vartak, Gudrun Wibbelt, et al.. Intravital dynamic and correlative imaging reveals diffusion-dominated canalicular and flow-augmented ductular bile flux. Hepatology, 2021, 73 (4), pp.1531-1550. 10.1002/hep.31422 . hal-03135253

\section{HAL Id: hal-03135253 \\ https://hal.inria.fr/hal-03135253}

Submitted on 8 Feb 2021

HAL is a multi-disciplinary open access archive for the deposit and dissemination of scientific research documents, whether they are published or not. The documents may come from teaching and research institutions in France or abroad, or from public or private research centers.
L'archive ouverte pluridisciplinaire HAL, est destinée au dépôt et à la diffusion de documents scientifiques de niveau recherche, publiés ou non, émanant des établissements d'enseignement et de recherche français ou étrangers, des laboratoires publics ou privés. 
Article type : Original

\section{Intravital dynamic and correlative imaging reveals diffusion-dominated canalicular and flow-augmented ductular bile flux}

Authors: Nachiket Vartak ${ }^{1 *}$, Georgia Guenther ${ }^{1 \#}$, Florian Joly ${ }^{2 \#}$, Amruta Damle-Vartak ${ }^{1}$, Gudrun Wibbelt ${ }^{8}$, Jörns Fickel ${ }^{8,10}$, Simone Jörs ${ }^{4}$, Brigitte Begher-Tibbe ${ }^{1}$, Adrian Friebel ${ }^{3}$, Kasimir Wansing ${ }^{3}$, Ahmed Ghallab ${ }^{1,7}$, Marie Rosselin ${ }^{5}$, Noemie Boissier ${ }^{2}$, Irene VignonClementel $^{2}$, Christian Hedberg ${ }^{5}$, Fabian Geisler ${ }^{4}$, Heribert Hofer ${ }^{8,9}$, Peter Jansen ${ }^{6}$, Stefan Hoehme $^{3}$, Dirk Drasdo ${ }^{2,1}$, Jan G. Hengstler ${ }^{1 *}$

\section{Affiliations:}

${ }^{1}$ Leibniz Research Centre for Working Environment and Human Factors (IfADo), Dortmund, Germany

${ }^{2}$ Inria, Paris, France

${ }^{3}$ Institute for Computer Science, Leipzig, Germany

${ }^{4}$ Klinikum rechts der Isar, Technical University Munich, Munich, Germany

${ }^{5}$ Umeå University, Umeå, Sweden

${ }^{6}$ Universiteit van Amsterdam, Amsterdam, Netherlands

${ }^{7}$ Faculty of Veterinary Medicine, South Valley University, Qena, Egypt.

${ }^{8}$ Leibniz Institute for Zoo and Wildlife Research (IWZ), Berlin, Germany

${ }^{9}$ Freie Universität Berlin, Berlin, Germany

${ }^{10}$ University of Potsdam, Potsdam-Golm, Germany

\# These authors contributed equally to the manuscript.

*Correspondence to: Nachiket Vartak, Email: vartak@bioimaging.tech

Jan G. Hengstler, Email: hengstler@ifado.de

This article has been accepted for publication and undergone full peer review but has not been through the copyediting, typesetting, pagination and proofreading process, which may lead to differences between this version and the Version of Record. Please cite this article as doi: 10.1002/HEP.31422

This article is protected by copyright. All rights reserved 


\section{Abstract (max 275 words):}

Small-molecule flux in tissue-microdomains is essential for organ function, but knowledge of this process is scant due to the lack of suitable methods. We developed two independent techniques that allow the quantification of advection (flow) and diffusion in individual bile canaliculi and in interlobular bile ducts of intact livers in living mice, namely Fluorescence Loss After Photoactivation (FLAP) and Intravital Arbitrary Region Image Correlation Spectroscopy (IVARICS). The results challenge the prevailing 'mechano-osmotic' theory of canalicular bile flow. After active transport across hepatocyte membranes bile acids are transported in the canaliculi primarily by diffusion. Only in the interlobular ducts, diffusion is augmented by regulatable advection. Photoactivation of fluorescein bis-(5-carboxymethoxy-2nitrobenzyl)-ether (CMNB-caged fluorescein) in entire lobules demonstrated the establishment of diffusive gradients in the bile canalicular network and the sink function of interlobular ducts. In contrast to the bile canalicular network, vectorial transport was detected and quantified in the mesh of interlobular bile ducts. In conclusion, the liver consists of a diffusion dominated canalicular domain, where hepatocytes secrete small molecules and generate a concentration gradient and a flow-augmented ductular domain, where regulated water influx creates unidirectional advection that augments the diffusive flux.

\section{One Sentence Summary (max 40 char)/Keywords:}

Bile flux proceeds by diffusion in canaliculi, augmented by advection in ducts. 


\section{Footnotes:}

\section{Contact information}

Nachiket Vartak

Imaging group - Dept. of Systems Toxicology

Lebniz Research Center for Working Environment and Human Factors (IfADo)

44139 Dortmund

Germany

Tel: +49-176-63031139

Email : vartak@bioimaging.tech

Jan G. Hengstler

Imaging group - Dept. of Systems Toxicology

Lebniz Research Center for Working Environment and Human Factors (IfADo)

44139 Dortmund

Germany

Tel: +48-231-1084-348

\section{Financial Support}

This study was supported by the Federal Ministry of Education and Research (BMBF) within the program Systems Medicine of the Liver (LiSyM) under award numbers 031L0055; 031L0045; and $031 \mathrm{~L} 0052$.

\section{Funding Statement}

Modeling studies were funded by the ANR under the project: iLite ANR-16-RHUS0005. 


\section{Main Text}

The flux of small molecules through tissue compartments and vessel networks is a fundamental process supporting organ function. Analysis of flux in microscopic vessels, cells and tissue compartments in living organisms remains intractable due to their inaccessibility to conventional rheological and ultrasonic methods. In this work, we used the liver as an exemplary organ and quantified transport in its microscopic biliary conduits. Liver tissue architecture consists of lobules

- functional units comprised of blood capillaries called sinusoids, hepatocytes, and a canalicular network formed by hepatocyte apical membranes (Fig 1A). The canalicular networks are linked to interlobular bile ducts (IBDs), which progressively converge into larger ducts and finally the extrahepatic bile duct (EHBD)(1).

The prevailing concept ascribes the movement of bile (solvent) and its constituent small molecule solutes (bile acids, xenobiotics etc.) to solvent fluid advection due to osmotic water influx and canalicular membrane contractility (2). Hepatocyte-mediated active transport of bile acids and other organic solutes into the canalicular lumen is expected to generate an osmotic gradient that drives the movement of water and causes flow of canalicular bile. Bile would then be pushed through the canalicular network with an increasing velocity towards IBDs, supported by peristalsis-like contractions of canalicular membranes (3). In the present study, we established Intravital Arbitrary Region Image Correlation Spectroscopy (IVARICS), fluorescence loss after photoactivation (FLAP) and time-lapse microscopy techniques to quantify flux in microdomains in live organisms. Applying these techniques to bile canaliculi and interlobular bile ducts revealed a diffusion-dominated canalicular and flow-augmented ductular domain through which bile flux occurs. We present a mechanistic model of bile flux that corroborates existing knowledge of bile clearance, but is not compatible with the prevailing mechano-osmotic concept of bile flow.

\section{Materials and Methods}

Materials and Methods for animal handling and preparation for microscopy, experimental details of intravital imaging procedures, acquisition parameters and analysis routines for photoactivation and IVARICS, in silico modeling, and synthesis of fluorescent bile acid analogs are provided in the Supporting Information. 


\section{Results}

Intravital analysis of bile flux was enabled by HNF1beta/CreER-reporter mice which express tdTomato specifically in cholangiocytes (4), and through the use of fluorescent analogs of bile salts. Fluorescent analogs such as cholyl-lysyl fluorescein (CLF) are transported by hepatocytes from sinusoids to canaliculi, allowing direct visualization of the canalicular network. Confocal or 2-photon imaging of live mouse livers show the canalicular networks and connecting interlobular bile ducts, depicting the basic lobular architecture in which biliary flux occurs (Fig. 1A).

\section{Determination of local flux mechanisms and the bounds of flux kinetics in the liver canalicular} network

Bile flux may occur either by advection or by inherent diffusion of the bile salts through the canalicular space, or a combination of both (convection). To investigate the local flux mechanisms, we utilized fluorescence loss after photo-activation (FLAP) of a photoactivatable analog of fluorescein (CMNB-Fluorescein) in intravital microscopy of mouse livers. Following tail vein injection, CMNB-Fluorescein (CMNB-Fluo) was photo-activated at time points when it was almost entirely localized in the canalicular network. Repeated photoactivation of CMNB-Fluo was performed in a pulsed manner on predefined regions of known spatial dimensions (Fig. 1B). By varying the time-interval between the photoactivation pulses, a race-condition is set up in the defined region between photoactivation pulses which increase fluorescence, and the underlying flux mechanism which depletes fluorescence. Accumulation of fluorescence with subsequent pulses indicates insufficiency of the underlying flux to remove the fluorescent material in the time interval between pulses. An upper-bound was established by decreasing the activation pulse rate until the underlying flux rate could finally remove the fluorescent material in the time-interval between activation pulses. Assuming pure advection in the canaliculi, the upper limit of the flux rate was determined to be $\mathbf{\sim 0 . 0 5} \boldsymbol{\mu m} / \mathbf{s}$ as the ratio of the maximum length of the activated region and the minimum interval between pulses in which it is depleted. Correspondingly, assuming pure diffusion, a lower bound of $\mathbf{0 . 5} \boldsymbol{\mu m}^{2} / \mathrm{s}$ is obtained for the diffusion coefficient from the ratio of the area of the activated region and the time-interval required to deplete fluorescence in the region. These limits of the flux rates of advection or diffusion suggest that the inherent diffusion of small molecule solutes such as bile acids dominates their transport, rather than advection of the solvent. 


\section{FLAP differentiates the diffusion-dominated and advection-augmented compartments of the}

\section{biliary system}

To determine the mechanism of bile flux, we examined the nature of the fluorescence loss in the photoactivated (FLAP) region after a single photoactivation pulse in a blood vessel, canalicular network and interlobular bile duct. The blood vessel represents a flow-dominated system, and thus the photoactivated mass disperses by molecular diffusion in the solvent, while moving along the with the vectorial blood flow (Fig. 1C, Mov. S1). We next measured the loss of fluorescence in a circular photoactivated region in the bile canalicular network, since the diameter of individual canaliculi approaches the optical resolution limit and is too small to precisely define a region within the lumen. The dispersion of photoactivated material is symmetric with a corresponding symmetric spread outside this region, irrespective of the lobular location of the activated zone (Fig 1C, Mov. S2). The location of the fluorescence maximum within the activated zone remains invariant over time. Alternative activation geometries (stripe, 3D) led to identical conclusions (Fig. S1A, Mov. S3, Mov. S10). This indicates that the local biliary flux in canaliculi is not vectorial but rather the dispersion of bile salts is symmetric and omnidirectional in the canalicular network. This implies molecular diffusion rather than vectorial flow as the primary flux mechanism in the canalicular network, consistent with the extremely low upper bound of pure advection in the canalicular network. The same intravital photoactivation technique was applied to intralobular bile ducts (Fig. 1C) that were specifically visualized through the cholangiocytespecific tdTomato-fluorescence-labeling in the HNF1beta/CreER-tdTomato mice. Contrary to observations in canaliculi but similar to blood, dispersion of fluorescence was asymmetrical with vectorial bias along one direction in the IBD. This indicates the presence of advection, in addition to diffusion, that conducts bile flux in the interlobular ducts. However, advection in the interlobular bile duct is substantially slower compared to the blood vessel.

To demonstrate diffusion-dominance in the canalicular network, we performed FLAP experiments at the Canal of Hering $(\mathrm{CoH})$ - connections of the canalicular network to IBDs. These junctional regions would represent the transition zone from the diffusion-dominated canalicular lumen to the advection-augmented ductular lumen. Photoactivated CMNB-Fluo in the canalicular network adjacent to the $\mathrm{CoH}$ (i.e. PV zone) transited to the IBD and diffused symmetrically into the network as expected (Fig. 2A). However, photoactivated CMNB-Fluo in the $\mathrm{CoH}$ also transited 
retrograde into the canalicular network (Fig. 2B). This retrograde flux of fluorescence from the $\mathrm{CoH}$ into the canalicular network confirmed that in the canalicular network, the inherent diffusion of small molecules overrules the meagre (if any) advection towards the bile duct.

We then confirmed that unidirectional flow in individual ductules (Fig. 1C) leads to a similar net vectorial flow in the larger ductular mesh surrounding portal veins. We photoactivated a region of the mesh encompassing several ductules, themselves further connected to other branch clusters. CMNB-Fluo preferentially migrated to only some of all available connected clusters of branches. This demonstrates vectorial flow in the larger interlobular biliary network (Fig. 2C).

The insignificant canalicular advection under basal conditions led us to test if conventional interventions that are known to increase extrahepatic bile flow could induce canalicular advection. Secretin is known to stimulate bile flow from the bile duct epithelium (5) and is expected to increase extrahepatic bile flow without increasing canalicular flux. In contrast, taurocholate (TCA) is excreted into canaliculi and expected to cause hyperosmotic water influx (6) to increase canalicular bile flow. Secretin as well as TCA infusion both induced an increase in extrahepatic bile flow from basal levels of $\sim 1 \mu 1 / \mathrm{min} / \mathrm{g}$ liver weight, upto $\sim 3 \mu 1 / \mathrm{min} / \mathrm{g}$ liver weight (Fig 3A), recapitulating previous reports $(7,8)$.

Photoactivation in various lobular zones and interlobular bile ducts was performed (Fig. 3B, Mov. S4) and the half-life of clearance was determined (Fig. S1B). Relative contributions of diffusion and advection were determined by quantification of the dispersion and displacement of Gaussian photo-activation profiles for the diffusion coefficient and velocity, respectively (9) (Supporting information: Photoactivation).

Under basal conditions, diffusion was similar in the interlobular ducts and all lobular zones of the canalicular network, ranging between median diffusion coefficients of $\mathbf{2 - 5} \boldsymbol{\mu m}^{2} / \mathbf{s}$ (Fig. 3C, Table S1). However, the advective velocity in canalicular networks was less than $\mathbf{0 . 0 2} \boldsymbol{\mu m} / \mathbf{s}$. In contrast, bile ducts showed an advective velocity ranging from 1-1.2 $\mu \mathrm{m} / \mathbf{s}$. These findings reinforce the deduction that biliary flux is diffusion-dominated in the canalicular network but show that it is augmented by advection in the bile ducts. Following secretin administration or TCA infusion, advection in the canalicular network did not significantly increase and the diffusion coefficient remained similar to that under basal conditions. In the interlobular bile ducts, the diffusion coefficient also remained unchanged from that of basal conditions, but the median advection velocity increased to up to $3.8 \mu \mathrm{m} / \mathrm{s}$ after secretin administration and up to $6.2 \mu \mathrm{m} / \mathrm{s}$ during 
TCA-infusion (Fig. 3C, Table S1). These findings are consistent with the known property of cholangiocytes to secrete water upon secretin stimulation (10) or when excess bile salts are detected in the bloodstream (11). Yet, even under these conditions, we find that the canalicular bile flux remains diffusion-dominated.

\section{Quantification of flux parameters for diffusion and advection - Intravital Arbitrary Region Image Correlation Spectroscopy (IVARICS)}

The quantification methods used for FLAP (9) assume a homogeneous fluid medium without diffusion barriers. Non-ideal conditions in a living mouse such as inherent inhomogeneity of the canalicular network, its finite size, boundary at the central vein and the sink at the CoH/IBD may affect the photo-activation profiles. To remove the effect of these non-ideal morphological constraints on determination of flux parameters, we established an orthogonal approach to determine diffusion and advection rates in the biliary compartments - namely, Intravital Arbitrary Region Image Correlation Spectroscopy (IVARICS). Image correlation spectroscopies (ICS) $(12,13)$ are based on rapid acquisition of fluorescence fluctuations caused by molecular movements (flux) in a defined region and the generation of corresponding correlation maps. The method is intrinsically local and yields apparent diffusion coefficients and advection velocities for the acquisition region. Image correlation techniques have distinct limitations from those of FLAP. They require a priori knowledge of the range in which flux parameters are expected, on which basis image acquisition and fitting parameters have to be set. In the case of in vivo imaging, light scattering by tissue and movements of the animal during image acquisition must be compensated for during analysis (see Supporting Information: IVARICS). Nonetheless, due to its fundamentally different imaging acquisition, analysis and limitations, IVARICS represents a complementary and orthogonal approach to FLAP analysis.

We first determined the feasibility of applying image correlation methods in vivo, by acquiring conventional spatial and temporal ICS sequences in the liver sinusoids, hepatocytes and canaliculi of mice infused with CLF. Since sinusoidal blood flow comprises both diffusion and advection, while the hepatocyte cytoplasm comprises only diffusion, these compartments served as in situ controls. IVARICS sequences could be used to extract the flux mechanism - sinusoidal blood flow was measured to be $\sim 60 \mu \mathrm{m} / \mathrm{s}$ and diffusion coefficients were $\sim 2 \mu \mathrm{m}^{2} / \mathrm{s}$ in all compartments (Fig. 4A). No advection could be detected in the hepatocyte cytoplasm as well as the canalicular lumen. 
The effect of animal movements due to heartbeat, respiration and intestinal peristalsis (Fig. S2, Mov. S5) could be mitigated during post-processing using pruning of slow autocorrelations and the use of the recently reported arbitrary region raster ICS (RICS) algorithm (13) (Fig. 4B, see Supporting Information). Spatial and temporal image autocorrelations (RICS/TICS) were independently fitted to appropriate functions (see Supporting Information: IVARICS) to determine diffusion coefficients and velocities (Fig. 3C, D, S2, Table S2, S3).

Diffusion coefficients and velocities derived from IVARICS analysis of CLF or fluoresceininfused mice confirmed the findings of the FLAP experiments (Fig. 3C, D, E). Median ductular advection velocity was measured to be $\sim 1 \mu \mathrm{m} / \mathrm{s}$ under basal conditions, which increased up to a value of $2.4 \mu \mathrm{m} / \mathrm{s}$ by the action of secretin on the duct, and up to $3.7 \mu \mathrm{m} / \mathrm{s}$ through TCA infusion (Fig. 4E). Flow in the canalicular network remained negligible and was neither affected by secretin administration, nor by TCA infusion. Diffusion coefficients in the canalicular network and interlobular ducts ranged between 2.4-6 $\mu \mathrm{m}^{2} / \mathrm{s}$. Thus, the general conclusion of the FLAP experiments was validated by IVARICS.

\section{Simulation of Bile Flux in the liver}

To validate if a system with diffusion-dominated canalicular flux, augmented by advection in the duct, is capable of accurately describing the behavior of bile acids in liver canalicular networks and ducts, we developed a deterministic Navier-Stokes in silico model in realistic canalicular geometries of the liver tissue. Confocal scans of immunofluorescence stained liver tissue showing canalicular network and interlobular bile ducts were generated as described previously (14) and digitized into triangulated meshes that served as the simulation space (Fig. 5A). Concentrations of fluorescein or CLF generated within various liver compartments during our experiments were estimated by establishing instrument calibration curves in murine bile in vitro (Fig. S3A). These concentrations, and the diffusion coefficients as well as velocities for the canalicular network and IBDs determined thus far were used as input parameters for the simulations.

Simulations of repeated activation as described earlier (Fig. 1B) quantitatively reproduced the upper-bound of flux rates (Fig. 5B). Simulations of single photoactivations in the canalicular network reproduced symmetric canalicular dispersion and unidirectional displacement of intensity in the IBD (Fig. 5C) as was observed experimentally (Fig. 1C). The retrograde flux of 
fluorescence into the canalicular network from the $\mathrm{CoH}$ (Fig. 1D) was also accurately predicted by the simulations (Fig. 5D). The half-times of depletion in silico for a given area were similar to those observed in vivo (Fig. 5C, Fig. S1B), and varied proportionately to the area of photoactivation. In summary, the 'first-principles' simulation accurately recapitulated all experimental observations for using experimentally derived input parameters for diffusion and advection in the various zones. Thus, the simulation reinforced our findings of a diffusiondominated canalicular network with negligible (if any) advection and an advection-augmented ductular system.

\section{Factors (not) influencing global canalicular clearance rates}

Water influx due to the osmotic gradient created by bile salts, wherein the cholic acid moiety represents the osmotically-active moiety, is the presumed raison d'etre for canalicular advection. Since our results so far indicated no such advection in the canalicular network, we addressed the effect of osmosis on global clearance of the bile salt analog cholyl-lysyl-fluorescein (CLF) from the liver lobule under basal or stimulated extrahepatic bile flow (TCA infusion, secretin administration) conditions. In particular, the effect of TCA to increase extrahepatic bile flow is ascribed to increased canalicular flow due to the aforementioned osmotic gradient (2).

Following intravenous injection of CLF into the tail-vein, intravital time-lapse microscopy showed that the rate of CLF clearance from the canalicular network was unchanged between basal conditions or secretin- and TCA-stimulated conditions (Fig. 6A, B, D, Mov. S6). To rule out the possibility that the CLF molecule itself created a strong, perhaps maximal, osmotic gradient, we performed calibrated quantitative comparison of canalicular excretion of its fluorophore (fluorescein) with and without co-injection of its osmotically active component, cholic acid (15). Co-injection of cholic acid along with fluorescein did not significantly alter the kinetics of fluorescein excretion from the canalicular network (Fig. 6C, D, Mov. S7). These observations are incompatible with a role of osmosis in canalicular excretion. Since the 'difference in osmotic potential' assumption could not explain the differences in the half-life of clearance of CLF and fluorescein, we hypothesized that intrinsic transporter activity of the hepatocyte generates their different clearance kinetics. CLF and fluorescein differ in molecular size, structure and transporter specificity $(16,17)$ and hence direct comparisons between the two compounds as substrates for transporter activity are complex. We therefore synthesized cholic acid derivatives CL-ATTO405 
and CL-ATTO565, conjugated with 2 spectrally distinct, but chemically similar fluorophores. Coinjection of both compounds into the tail vein showed that hepatocyte export of CL-ATTO405 occurred at a much higher rate than that of CL-ATTO565. This shows that the transporter activity differs between the two compounds. However, in both cases, canalicular depletion closely followed the hepatocyte export kinetics. Thus, the higher export of CL-ATTO405 did not saturate the diffusive canalicular flux. Rather, hepatocyte export was the rate limiting step for both compounds, and canalicular intensity remained correlated to the intensity in the hepatocytes (Fig. S3C, Table S4). Taken together, these results show that for bile acid concentrations of up to 400 $\mu \mathrm{M}$ (calibration curve, Fig. S3A) the hepatocyte export activity, rather than osmotic potential, determines canalicular clearance rates of bile acid analogs.

\section{Visualization of the lobule-wide diffusion gradient}

A diffusive system such as the canalicular network should establish higher concentrations at the closed (pericentral) end than at the sink (periportal) end. Such a gradient is a necessary condition to drive diffusive flux out of the network. However, local concentrations in canaliculi depend not only on canalicular flux, but also on hepatocyte export. To investigate the influence of these factors, we measured the lobule-wide spatiotemporal distribution and clearance of carboxymethylfluorescein diacetate (CMFDA). Intracellular esterases cleave acetate groups from the nonfluorescent CMFDA which enters hepatocytes to generate the 400-fold brighter fluorescein molecule, which is excreted then into the canaliculi. Following tail-vein injection, fluorescein appears earlier and with higher intensity in periportal than in pericentral canaliculi (Fig. 7A, Movie S8). Within approximately an hour, the intensities in the periportal and pericentral canaliculi equilibrate to similar levels. These experiments show that the canalicular fluorescein excretion by hepatocytes occurs in a PV-CV gradient. This transport-imposed gradient would antagonize and mask a diffusion-derived CV-PV canalicular gradient. To compare the relative clearance rates in periportal versus pericentral canaliculi, temporal intensity profiles were normalized to their maximal values. Normalized profiles showed a higher depletion rate in periportal than pericentral canaliculi, hinting at a diffusion-mediated gradient masked by an antidromic transport gradient.

To demonstrate the existence of this diffusion-gradient, we used a method that allows the sole analysis of the influence of diffusion on the canalicular intensity, without the interference of the 
hepatocellular export. For this purpose, we photoactivated CMNB-Fluo lobule-wide approximately 1 hour after tail-vein injection, when the material is predominantly in the canaliculi and not in the hepatocyte cytoplasm. Immediately after photoactivation, a CV-PV gradient in canalicular intensity was visible which steepened over time (Fig.7B, Movie S9). After normalization to maximal intensities, the faster clearance rate of the periportal compared to pericentral canaliculi became even more apparent. Taken together, we conclude that diffusion indeed establishes a CV-PV canalicular gradient, but which is antagonized by higher hepatocellular export in periportal zone.

\section{Discussion and Conclusion}

In this work, we combined advanced intravital imaging with simulation techniques in real geometries to investigate the flux characteristics of tissue microdomains. IVARICS is an adaptation of image correlation spectroscopy, wherein the decay in correlation between pixels due to fluctuation of the signal created by molecular flux is used to compute the rate of flux, with specialized corrections for periodic animal movements caused by its heartbeat, respiration and peristalsis. This allowed us to quantitatively decipher the mode of transport and determine its rate for small molecules in a sub-micron scale vessel network of arbitrary geometry. Utilizing FLAP studies, correlative imaging, rheological measurements and custom chemical probes, we have addressed a specific long-standing problem in liver physiology.

Diffusion-dominated canalicular bile flux contradicts the concept of advective bile flow in the canaliculi that has been reported in medical text books since decades. The osmotic theory of bile flux states that bile flows through the canaliculi countercurrent to the direction of sinusoidal blood flow, due to osmolytes including bile acids being actively exported into the canalicular lumen, simultaneously drawing water along the osmotic gradient (2). Since the canalicular network is closed at the pericentral end, water influx results in a unidirectional flow towards the bile duct. While the theoretical concept of the osmotic theory was proposed already in 1959 (18), corroboratory experiments were presented in the following decades, comprehensively reviewed by Boyer (2). A general feature of these experiments is the intravenous administration of compounds such as bile salts $(8,19-22)$ with simultaneous measurement of increased extrahepatic bile volume and biliary compound excretion. Since bile acids and other organic solutes are secreted by hepatocytes into the bile canaliculi, and extrahepatic bile flow varied approximately linearly with 
amount of infused compound, it was concluded that the canaliculi are the anatomical structure where flow is initiated. Further, because the apparently linear relationship seemed to contain an inherent offset, the resultant extrahepatic bile flow was proposed to consist of a bile-salt dependent fraction which varies with the amount of administered bile acid, and a bile-salt independent fraction attributed to other osmolytes than bile acids, and/or to water secretion by cholangiocytes (2). As long as the focus is on macro-scale correlations between the infusion and clearance of compounds from blood and the excreted volume of extrahepatic bile as well as the excreted amounts of compounds, the osmotic flow concept creates no contradiction with experiment.

Yet, it has also been recognized early on that the postulated linear relationship between infused compounds and extrahepatic bile flow is only approximate $(18,19,22-24)$. This unsatisfactory linearity has been dealt with by introducing a 'correction for delay in transit in the biliary tree' when comparing two compounds (22). Nahrwold and Grossman (24) detected threshold effects in the induction of increased extrahepatic bile flow - an observation incompatible with the osmotic theory without further assumptions. Wheeler (19) specifically recognizes through theoretical considerations that diffusion could also explain the experimentally observed bile salt excretion, and laments that direct canalicular flow measurements are not available. In later work (25), Wheeler and Ramos cautiously state that the osmotic theory "would provide an adequate explanation" but agree that for such observations, it does "not seem necessary to invoke a specific mechanism for active water transport." Indeed, as long as the focus is on macro-scale extrahepatic bile flow, the precise mechanism of canalicular transport (diffusion/advection/convection) is not relevant in order to derive these correlations. However, this mechanism becomes relevant for other questions such as the influence of pathological biliary pressure in canaliculi or the site of action of choleretic drugs.

For example, the concept of 'osmotic canalicular bile flow' has been used to mathematically model the velocity of canalicular bile flow and the resulting pressure, claiming a bile velocity in periportal canaliculi of up to $\sim 12 \mu \mathrm{m} / \mathrm{s}$ in mice (3). Based on the osmotic concept of bile flow, another study simulated pericentral canalicular pressure in individual patients with NASH and calculated increases above $3000 \mathrm{~Pa}$ due to altered canalicular morphology. This was interpreted 'as a new component of the NAFLD pathophysiology' (26). The applicability of these models crucially depends on what the actual molecular mechanisms of canalicular flux are. Leaving such 
conclusions (26) unchallenged may encourage the development of unfounded therapies that focus on modifying canalicular flow and pressure.

To our knowledge, no previous study in these intervening 40 years has measured flow and diffusion in bile canaliculi and interlobular bile ducts. Therefore, the part of the osmotic theory that bile flows countercurrent to sinusoidal blood in the canaliculi remained untested. In the present study, we directly investigated the flux mechanism in these microdomains and exclude the canaliculi as the anatomical site where flow originates. The osmotic concept did not consider that the transport of solutes may also occur through a standing water compartment by molecular diffusion. The techniques developed in our study allow to experimentally quantify flow and diffusion in bile canaliculi and ducts of intact livers in situ, and thus to validate or falsify previous model predictions.

Our results show that bile acids are transported primarily through diffusion in the canaliculi, while being augmented by directional advection in the interlobular ducts. Through these observations, we deduced the compartmentalization of the liver into a diffusion-dominated canalicular domain where hepatocytes actively secrete small molecules and generate a concentration gradient that drives diffusive flux towards the IBDs (Fig. 8A). In IBDs, regulated and inducible water influx creates unidirectional advection which augments the diffusive flux. Water influx into ducts can be caused either directly by hormones such as secretin (27), or indirectly by bile salts themselves as demonstrated for TCA (10), explaining the corresponding increase in extra-hepatic bile flow. This inducible increase in advection is restricted to the bile ducts, while the canalicular network remains nothing more than a standing-water zone.

We note that the principle of liver physiology presented in this work, with a diffusion-dominated standing-water domain connected to an inducible flow-augmented excretion duct, seems a general principle applicable to exocrine glands (Fig. 8A, B). All exocrine glands contain an acinus, surrounded by epithelial cells that secrete their products into the acinar lumen. The lumen represents a reservoir where products accumulate to high concentrations, which is connected to an excretion duct in which flow can be induced on demand. A similar design enables the function of all $\sim 2$ types of mammalian exocrine glands (28), of which the liver is the largest. For example, lactating mammary glands synthetize milk proteins consistently in the acini, but secretion of milk is induced through the action of hormones such as prolactin on the mammary ducts. From this perspective, the canalicular network may be envisaged as a highly reticulated form of the 
conventional spheroidal acinus (Fig. 8B). The reticulated geometry is probably imposed due to the necessity of hepatocytes to maximize their contact area with the also reticulated sinusoidal network, which dictates their positioning. This modified acinus is connected to flow-augmented bile ducts of the liver, following the general principle of exocrine glands.

The reticulated acinus (bile canaliculi) of the liver implies reduced degrees of freedom for molecular flux, compared to a spherical acinus and contributes to complex zonation phenomena. Due to diffusion, a gradient with higher bile acid concentrations at the pericentral closed end of bile canaliculi is established, while concentrations at the periportal open end are lower (Fig. 7D, upper panel). However, the diffusive CV-PV gradient is antagonized by an antidromic gradient, caused by the higher canalicular excretion rate of PV than PC hepatocytes (Fig. 7E, lower panel). The preferential PV excretion of bile acids and other compounds is in agreement with previous studies, injecting $\left[{ }^{3} \mathrm{H}\right]$ taurocholate into the portal vein of isolated perfused rat livers, which led to a steep PV-CV gradient of radioactive tracer in liver tissue (29). Vice versa, the same authors also show that retrograde perfusion with $\left[{ }^{3} \mathrm{H}\right]$ taurocholate injection into the central vein led to a CV-PV gradient (30). These experiments suggest that uptake from the sinusoid into hepatocytes depends on the sinusoidal blood concentration that decreases during passage through the lobule. As the excretion and the diffusion driven gradients are antidromic (Fig. 8C), it is difficult to visualize the diffusive gradient by the use of conventional fluorescent 'always-on' tracers such as CLF or CMFDA. However, the diffusive CV-PV gradient can be demonstrated by photoactivatable fluorophores, e.g. CMNB-Fluo, if photoactivation is performed when most of the compound has been excreted to the canaliculi. Preferential excretion of bile acids in the PV zone leads to diffusive equilibration into the duct, but also rest of the canalicular network towards the CV zone. This retrograde loading means that bile acids are kinetically-trapped in the network at a relatively high concentration. In case of a demand of bile acids in the intestine after food intake, low $\mathrm{pH}$ in the stomach (and duodenum) triggers S-cells in the mucosa of the duodenum to release secretin into the blood, which causes water influx into bile ducts via cholangiocytes and thereby flow of bile to the gall bladder, which releases its content into the duodenum under the control of other hormones, such as cholecystokinin(31). Therefore, hepatocytes represent a domain of continuous bile acid synthesis, canaliculi serve as an intermediate store, while the duct acts as a logistic center that serves the food intake dependent needs of the intestine for bile acids. Photoactivation in the present study was performed for small regions, (e.g. radius $=10 \mu \mathrm{m}$ ), to analyze local flux properties, as well as for large areas comprising entire lobular sections (radius $=200 \mu \mathrm{m}$ ). These 
experiments clearly demonstrated that the diffusion-dominated flux in canaliculi, and advectionaugmented flux in ducts holds at the scale of the lobule. Diffusion gradients could be demonstrated over the lobular canalicular network, while vectorial transport was observed in the mesh of interlobular bile ducts around portal veins. Photoactivation of entire lobules also demonstrated the sink function of interlobular duct meshes since canalicular intensities decreased faster in the neighborhood of these structures, while fluorescent material was cleared more slowly in the pericentral zone. It should be considered that a flow-dominated mechanism would create a gradient of the opposite orientation with lower concentrations in the lobule center (CV zone).

Why does the excretion of bile acids into the canaliculi, does not cause any measurable advection? Advection is not the inevitable result of the transport of osmotically active compounds into a biological network with a closed end. Bile acids, as hydrophobic molecules, are incorporated in phospholipid micelles, preventing them from exerting their full osmotic potential (15). Rather, the effect of inorganic ionic balance is clearly known to play a complex role in bile acid excretion and extrahepatic bile flow $(25,32)$. Yet, the secretion of bile acids by hepatocytes will necessitate simultaneous uptake of anions such as $\mathrm{Cl}^{-}$to maintain plasma membrane potential (33). Homeostatic ion exchange may counteract the development of any net osmotic pressure in the canalicular lumen. The present analyses show that advection does not exceed $0.02 \mu \mathrm{m} / \mathrm{s}$ in the canalicular network, suggesting that as far as a flow is concerned, the osmotic effect of osmotic ion exchange is exerted primarily in the biliary ducts - not the canalicular network.

Canaliculi are known to contract autonomously and asynchronously at a low frequency of 6 events/hour through a calcium-dependent mechanism (34). This has been proposed to cause propulsion of bile in the canaliculi. Again, the absence of any significant advection in the canalicular network indicates that in the absence of synchronized contractions along the CV-PV axis, merely mixing (rather than pumping) of canalicular contents may occur.

One of the surprising aspects of flux in the canalicular network is the low diffusion coefficient of $2.4-4.6 \mu \mathrm{m}^{2} / \mathrm{s}$ (CI: 95\%), compared to $\sim 200 \mu \mathrm{m}^{2} / \mathrm{s}$ in water in vitro (Fig. S3B). Biliary constituents such as albumin and phospholipid micelles may interact with bile acids to reduce their diffusivity. The apparent value of a diffusion coefficient may represent anomalous diffusion processes such as (i) polydispersive diffusion (35) - the convolved diffusivity and binding interactions of multiple populations ranging from micellar to freely diffusing bile acid molecules, or (ii) Knudsen diffusion - due to the relatively small size of the diffusible volume compared to the 
mean path length. Nonetheless, even the relatively low apparent diffusion coefficient is sufficient to account for observed lobular clearance rates, as verified by the diffusion simulation in realistic canalicular geometries. Moreover, we show that hepatocyte cytoplasmic concentration is the strongest factor of influence of canalicular concentrations for bile salt analogs over the entire clearance period (Fig. S3C). Thus, we corroborate previous reports $(36,37)$, that energy-dependent export from hepatocytes to the canalicular lumen is a stringent bottleneck for bile acid clearance. Diffusive flux through the canalicular network in not limiting. Due to high periportal transport rates, high concentrations of bile acids are created near the interlobular bile ducts, reducing the distance bile acids have to cross by diffusion. Effectively, the functional diffusive zone may be smaller than the actual anatomical size of the lobule.

In summary, this work demonstrates the flux mechanisms that operate in the various liver microdomains and provides a generalized methodology to perform quantitative intravital evaluation of flux-associated organ function. The liver follows the principle of parsimony - the energy invested in active transport across membranes is entropically dissipated for canalicular diffusion, to be augmented by energy-driven regulated flow in the ducts as required for physiology.

\section{Author contributions}

Conceptualization, Methodology, NV, JGH, DD, FJ, CH; Investigation, NV, GG, FJ, MR; Writing - Original draft, NV, JGH, DD; Writing - Review and Editing, NV, JH, JF, DD; Resources, FG, SJ, GW, HH; Funding acquisition, JH, DD, NV; Data curation, Validation, JF, BBT, AG, KW, AF, SH, IVC, NB; Supervision, Project administration, JH, DD, NV, HH, SH, CH.

\section{References}

1. Vartak N, Damle-Vartak A, Richter B, Dirsch O, Dahmen U, Hammad S, et al. Cholestasisinduced adaptive remodeling of interlobular bile ducts. Hepatology. 2016;63:951-964.

2. Boyer JL. Bile formation and secretion. Compr. Physiol. 2013;3:1035-1078. 
3. Meyer K, Ostrenko O, Bourantas G, Morales-Navarrete H, Porat-Shliom N, SegoviaMiranda F, et al. A Predictive 3D Multi-Scale Model of Biliary Fluid Dynamics in the Liver Lobule. Cell Syst. 2017;4:277-290.e9.

4. Jörs S, Jeliazkova P, Ringelhan M, Thalhammer J, Dürl S, Ferrer J, et al. Lineage fate of ductular reactions in liver injury and carcinogenesis. J. Clin. Invest. 2015;125:2445-2457.

5. Afroze S, Meng F, Jensen K, McDaniel K, Rahal K, Onori P, et al. The physiological roles of secretin and its receptor. Ann. Transl. Med. 2013;1:29.

6. Saeki T, Takahashi N, Kanamoto R, Iwami K. Characterization of Cloned Mouse $\mathrm{Na}+$ /taurocholate Cotransporting Polypeptide by Transient Expression in COS-7 Cells. Biosci. Biotechnol. Biochem. 2002;66:1116-1118.

7. Bodewes FAJA, Bijvelds MJ, Vries W de, Baller JFW, Gouw ASH, Jonge HR de, et al. Cholic Acid Induces a Cftr Dependent Biliary Secretion and Liver Growth Response in Mice. PLOS ONE. 2015;10:e117599.

8. Boyer JL, Scheig RL, Klatskin G. The effect of sodium taurocholate on the hepatic metabolism of sulfobromophthalein sodium (BSP). The role of bile flow. J. Clin. Invest. 1970;49:206-215.

9. Kang M, Day CA, Kenworthy AK, DiBenedetto E. Simplified equation to extract diffusion coefficients from confocal FRAP data. Traffic. 2012;13:1589-1600.

10. Alpini G, Glaser SS, Ueno Y, Rodgers R, Phinizy JL, Francis H, et al. Bile acid feeding induces cholangiocyte proliferation and secretion: Evidence for bile acid-regulated ductal secretion. Gastroenterology. 1999;116:179-186.

11. Alpini G, Ueno Y, Glaser SS, Marzioni M, Phinizy JL, Francis H, et al. Bile acid feeding increased proliferative activity and apical bile acid transporter expression in both small and large rat cholangiocytes. Hepatology. 2001;34:868-876.

12. Digman MA, Gratton E. Scanning image correlation spectroscopy. Bioessays News Rev. Mol. Cell. Dev. Biol. 2012;34:377-385. 
13. Hendrix J, Dekens T, Schrimpf W, Lamb DC. Arbitrary-Region Raster Image Correlation Spectroscopy. Biophys. J. 2016;111:1785-1796.

14. Damle-Vartak A, Begher-Tibbe B, Gunther G, Geisler F, Vartak N, Hengstler JG. Pipe-3D: A Pipeline Based on Immunofluorescence, 3D Confocal Imaging, Reconstructions, and Morphometry for Biliary Network Analysis in Cholestasis. Methods Mol. Biol. Clifton NJ. 2019;1981:25-53.

15. Carpenter P, Lindenbaum S. Osmotic and activity coefficients of aqueous bile salt solutions at 25,37 , and $45^{\circ}$ C. J. Solut. Chem. 1979;8:347-357.

16. de Waart DR, Häusler S, Vlaming MLH, Kunne C, Hänggi E, Gruss H-J, et al. Hepatic transport mechanisms of cholyl-L-lysyl-fluorescein. J. Pharmacol. Exp. Ther. 2010;334:7886.

17. Wilton JC. Fluorescent choleretic and cholestatic bile salts take different paths across the hepatocyte: transcytosis of glycolithocholate leads to an extensive redistribution of annexin II. J. Cell Biol. 1994;127:401-410.

18. Sperber I. Secretion of Organic Anions in the Formation of Urine and Bile. Pharmacol. Rev. 1959;11:109-134.

19. Wheeler H, Ross E, Bradley S. Canalicular bile production in dogs. Am. J. Physiol.-Leg. Content. 1968;214:866-874.

20. Wood RAB, Baker AL, Hall AW, Boyer JL, Moossa AR. Evaluation of a New Monkey Model for the Repeated Study of Bile Secretory Physiology: Ann. Surg. 1977;185:349-355.

21. Layden TJ, Elias E, Boyer JL. Bile formation in the rat: the role of the paracellular shunt pathway. J. Clin. Invest. 1978;62:1375-1385.

22. Boyer JL, Bloomer JR. Canalicular Bile Secretion in Man STUDIES UTILIZING THE BILIARY CLEARANCE OF [14C]MANNITOL. J. Clin. Invest. 1974;54:773-781.

23. Preisig R, Cooper HL, Wheeler HO. THE RELATIONSHIP BETWEEN TAUROCHOLATE SECRETION RATE AND BILE PRODUCTION IN THE 
UNANESTHETIZED DOG DURING CHOLINERGIC BLOCKADE AND DURING SECRETIN ADMINISTRATION*. J. Clin. Invest. 1962;41:1152-1162.

24. Nahrwold DL, Grossman MI. Secretion of Bile in Response to Food with and Without Bile in the Intestine. Gastroenterology. 1967;53:11-17.

25. Wheeler HO, Ramos OL. DETERMINANTS OF THE FLOW AND COMPOSITION OF BILE IN THE UNANESTHETIZED DOG DURING CONSTANT INFUSIONS OF SODIUM TAUROCHOLATE*. J. Clin. Invest. 1960;39:161-170.

26. Segovia-Miranda F, Morales-Navarrete H, Kücken M, Moser V, Seifert S, Repnik U, et al. Three-dimensional spatially resolved geometrical and functional models of human liver tissue reveal new aspects of NAFLD progression. Nat. Med. 2019;25:1885-1893.

27. Lenzen R, Alpini G, Tavoloni N. Secretin stimulates bile ductular secretory activity through the cAMP system. Am. J. Physiol. 1992;263:G527-32.

28. Wang J, Laurie GW. Organogenesis of the exocrine gland. Dev. Biol. 2004;273:1-22.

29. Groothuis GM, Hardonk MJ, Keulemans KP, Nieuwenhuis P, Meijer DK. Autoradiographic and kinetic demonstration of acinar heterogeneity of taurocholate transport. Am. J. Physiol. 1982;243:G455-462.

30. Groothuis GM, Meijer DK. Hepatocyte heterogeneity in bile formation and hepatobiliary transport of drugs. Enzyme. 1992;46:94-138.

31. Kanno N, LeSage G, Glaser S, Alpini G. Regulation of cholangiocyte bicarbonate secretion. Am. J. Physiol.-Gastrointest. Liver Physiol. 2001;281:G612-G625.

32. Bear CE, Strasberg SM. Techniques for Studying Biliary Secretion: Electrolytes in Bile. Hepatology. 1984;4:25S-30S.

33. Claret B, Claret M, Mazet JL. Ionic transport and membrane potential of rat liver cells in normal and low-chloride solutions. J. Physiol. 1973;230:87-101. 
34. Gupta K, Li Q, Fan J, Li Shan Fong E, Song Z, Mo S, et al. Actomyosin Contractility Drives Bile Regurgitation as an Early Homeostatic Response to Increased Biliary Pressure in Obstructive Cholestasis. BioRxiv [Internet]. 2016 [cited 2016 Oct 23];Available from: http://biorxiv.org/lookup/doi/10.1101/077792

35. Kalwarczyk T, Kwapiszewska K, Szczepanski K, Sozanski K, Szymanski J, Michalska B, et al. Apparent Anomalous Diffusion in the Cytoplasm of Human Cells: The Effect of Probes' Polydispersity. J. Phys. Chem. B. 2017;121:9831-9837.

36. Trauner M. Molecular alterations of canalicular transport systems in experimental models of cholestasis: possible functional correlations. Yale J. Biol. Med. 1997;70:365-378.

37. Geier A, Wagner M, Dietrich CG, Trauner M. Principles of hepatic organic anion transporter regulation during cholestasis, inflammation and liver regeneration. Biochim. Biophys. Acta. 2007;1773:283-308.

38. Jeliazkova P, Jörs S, Lee M, Zimber-Strobl U, Ferrer J, Schmid RM, et al. Canonical Notch2 signaling determines biliary cell fates of embryonic hepatoblasts and adult hepatocytes independent of Hes1. Hepatology. 2013;57:2469-2479.

39. Reif R, Ghallab A, Beattie L, Günther G, Kuepfer L, Kaye PM, et al. In vivo imaging of systemic transport and elimination of xenobiotics and endogenous molecules in mice. Arch. Toxicol. 2017;91:1335-1352.

40. Javitt NB, Emerman S. Effect of sodium taurolithocholate on bile flow and bile acid excretion. J. Clin. Invest. 1968;47:1002-1014.

41. Kountouras J, McKavanagh S, Burmicky M, Billing BH. The effect of secretin on bile flow and bile acid and bilirubin excretion following relief of prolonged bile duct obstruction in the rat. J. Hepatol. 1987;4:198-205.

42. Andresen V, Pollok K, Rinnenthal J-L, Oehme L, Günther R, Spiecker H, et al. HighResolution Intravital Microscopy. PLoS ONE [Internet]. 2012 [cited 2019 Sep 5];7. Available from: https://www.ncbi.nlm.nih.gov/pmc/articles/PMC3522675/ 
43. Kolin DL, Ronis D, Wiseman PW. k-Space Image Correlation Spectroscopy: A Method for Accurate Transport Measurements Independent of Fluorophore Photophysics. Biophys. J. 2006;91:3061-3075.

44. Rossow MJ, Mantulin WW, Gratton E. Scanning laser image correlation for measurement of flow. J. Biomed. Opt. 2010;15:026003.

45. Hebert B, Costantino S, Wiseman PW. Spatiotemporal image correlation spectroscopy (STICS) theory, verification, and application to protein velocity mapping in living $\mathrm{CHO}$ cells. Biophys. J. 2005;88:3601-3614.

46. Weller HG, Tabor G, Jasak H, Fureby C. A tensorial approach to computational continuum mechanics using object-oriented techniques. Comput. Phys. 1998;12:620-631.

47. Roache PJ, Ghia KN, White FM. Editorial Policy Statement on the Control of Numerical Accuracy. J. Fluids Eng. 1986;108:2. 


\section{Figure Legends}

Fig. 1. Definition of diffusion-dominated and advection-augmented compartments of the biliary system and identification of the upper-bound of flux kinetics. A. The diagram depicts the microdomains analyzed in the present study connected to macroscopic structures of the downstream biliary tract. Confocal maximum intensity projection $(20 \mu \mathrm{m})$ of HNF1beta/CreERtdTomato labeled bile ducts (red) and CLF-enriched canalicular network and duct lumen (green) 10 min after tail-vein injection of CLF in live mice. White dotted line indicates the portal vein. B. Representative sequence of repeating CMNB-Fluo (CMNB-Fluo) photoactivation, with pulses spaced $1.6 \mathrm{~s}$ or $223 \mathrm{~s}$ apart, showing accumulating fluorescence due to insufficient depletion of a $10 \mu \mathrm{m} \times 10 \mu \mathrm{m}$ activation region by canalicular bile flux. The graph shows quantification of intensities, indicating that CMNB-Fluo requires $>40$ s to be cleared from a $10 \mu \mathrm{m} \times 10 \mu \mathrm{m}$ region by canalicular flux. C. Photoactivation of CMNB-Fluo-Dextran $(10 \mathrm{Kd})$ in a central vein blood vessel and CMNB-Fluo in the canalicular network and duct showing the activated region (white circle) with its centroid (red, AC), and the distance travelled by the fluorescence fronts (F1, F2) from the centroid due to bile flux. Ducts show vectorial flux $(\mathrm{F} 1>\mathrm{F} 2)$ in contrast to symmetric dispersion in the canalicular network $(\mathrm{F} 1 \sim \mathrm{F} 2)$. The upper panel shows raw images (green: CMNBFluo, red: tdTomato marking ducts) while the lower panel represents the same with a polychromatic color table and annotations. Scale bars: $20 \mu \mathrm{m}$. The graphs depict the temporal evolution of spatial intensity profiles after photoactivation, with the intensity centre-of-mass drifting in IBDs but a remaining static in the canalicular network. Photoactivated region: White dotted circle. Scale bar: $20 \mu \mathrm{m}$.

Fig. 2. Demonstration of bile acid flux within and between diffusion-dominated canaliculi network and advection-augmented ductular mesh

A. Representative images of photoactivation in the PV zone adjacent to a $\mathrm{CoH}$ junction. B. Photoactivation in the $\mathrm{CoH}$ junction (right) showing ductular transit and retrograde flux into the canalicular network, respectively. Duct and $\mathrm{CoH}$ are annotated as dotted lines. C. Photoactivation in a ductular mesh surrounding a portal vein. Clusters of branches of the ductular network are indicated. Photoactivated region: White dotted circle. Scale bar: $20 \mu \mathrm{m}$.

\section{Fig. 3. Quantification of canalicular flux in basal and stimulated conditions}


A. Stimulation of extrahepatic bile flow after administration of a secretin bolus or TCA infusion. Stimulation was provided $30 \mathrm{~min}$ after extrahepatic bile collection started (arrow). Data indicated mean \pm S.D of 3 mice per condition. B. Representative time-lapse images following photoactivation in a defined region (white circle) of CMNB-Fluo (green) in canalicular networks adjacent to the central vein $(\mathrm{CV})$, portal vein $(\mathrm{PV})$ or in the mid-zone $(\mathrm{MZ})$, as well as in interlobular bile ducts marked by tdTomato (red). Scale bars: $50 \mu \mathrm{m}$. C. Quantification of diffusion coefficients from the half-life of intensity decay and advection velocity from shift of intensity centre-of-mass for various lobular zones (CV: pericentral; MZ: midzonal; PV: periportal) under basal conditions or stimulation with secretin and TCA. Values indicate measurements in $\mathrm{n}>8$ mice for each condition. Dots in the box plots represent the data of individual mice. ${ }^{* *} \mathrm{p}<0.01$ compared to controls.

Fig. 4. Establishment of IVARICS A. Representative images acquired for ICS analysis in canaliculi, hepatocyte cytoplasm and sinusoids. The graph shows quantification of diffusion coefficient and velocity in each compartment. B. Workflow of IVARICS analysis. Representative fluctuation images of fluorescein loaded canaliculi and ducts are acquired. A binary mask representing the structures of interest is created. Autocorrelation (ACF) maps are generated for both the intensity images and masks. A ratio of the intensity ACF and mask ACF yields a fluctuation ACF devoid of movement and shape effects. C. Average normalized spatial (y)autocorrelation for various liver domains with fits for 1-population 3D-diffusion. D. Average normalized temporal autocorrelation for various liver domains with fits for 1-population 3Ddiffusion and advection. E. Diffusion coefficients and velocities derived from spatial (RICS) and temporal (TICS) analysis for various canalicular zones (green) and interlobular bile ducts (red) under basal conditions or stimulation with secretin and TCA. Values indicate measurements of $n>4$ mice for each condition. The dots in the box plots represent the data of individual mice. $* * \mathrm{p}<0.01$ compared to controls.

Fig. 5. Simulation of bile acid flux mechanisms A. Representative 3D immunostained confocal stack (left) identifying the canalicular network and interlobular bile duct and its digitization to a polygonal mesh (right). B. Simulation of repeated photoactivation with various time intervals in a $10 \mu \mathrm{m}$ radius spherical region encompassing a canaliculus. Graphs show quantification of mean intensity in the activated spherical region with various activation intervals (compare: Fig. 1B). C. Simulation of single photoactivation in the canalicular network of varying radii (top, middle) and 
interlobular ducts (bottom), showing directional shift in the center of mass of the spatial intensity. Graphs show temporal profiles in the canalicular network (compare: Fig. S1B), spatial profiles in the IBD and canalicular network (compare: Fig. 1C). D. Simulation of photoactivation in the Canal of Hering, showing retrograde diffusion to the canalicular network (compare: Fig. 1D). All simulations were performed with experimentally derived flux parameters ( $D=3 \mu \mathrm{m}^{2} / \mathrm{s}, \mathrm{V}=0$ $\mu \mathrm{m} / \mathrm{s}$ in canaliculi, $1 \mu \mathrm{m} / \mathrm{s}$ in ducts). Photoactivated region: White dotted circle. Scale bar: $10 \mu \mathrm{m}$.

Fig. 6. TCA, secretin and cholate do not influence canalicular clearance A. Representative timelapse images of the canalicular clearance of CLF with and without TCA infusion. B. Representative time-lapse images of the canalicular clearance of CLF with and without secretin administration. C. Representative time-lapse images of the canalicular clearance of fluorescein with and without cholate injection. D. Quantitative comparison of clearance kinetics under conditions described in A, B, C, for $\mathrm{n}>6$ mice each, with no significant difference due to secretin, TCA or cholate treatment for the respective fluorophore. Scale bar: $50 \mu \mathrm{M}$.

Fig. 7. Visualization of lobule-wide bile flux gradients A. Representative time-lapse imaging of a liver lobule with tdTomato-positive interlobular ducts (IBD, red), infused with CMFDA (green). Bottom row shows false color images for visualization of CMFDA intensity. Graphs show raw and normalized fluorescence intensity indicating higher depletion rate in the periportal versus pericentral zones. Contour plot shows the hepatocyte-transport induced antidromic PV-CV gradient and diffusion-derived CV-PV gradient appear over time. B. Representative time-lapse imaging of a liver lobule with tdTomato-positive interlobular ducts (red), infused with CMNBFluo after photoactivation in the entire lobule. Bottom row shows false colour images for visualization of CMNBF intensity. Graphs show raw and normalized fluorescence intensity indicating higher depletion rate in the periportal versus pericentral zones. Contour plot shows the appearance of the diffusion-derived CV-PV gradient appear over time. Scale bar: $100 \mu \mathrm{m}$. CV indicates central vein.

Fig. 8. Flux organization in the liver A. Schematic representation of the diffusion-dominated domain, the canalicular network and the advection-augmented domain, the interlobular bile duct in the liver. Bile acids (BA) are secreted in the diffusion-dominated zone, while water is secreted in the advection-augmented zone. B. Comparison of liver tissue topography to that of a generic 
exocrine gland in terms of solute secretion zone and water secretion zone, implying that the liver canalicular network is analogous to an exocrine acinus, only modified geometrically from a sphere to a reticulated network. C. Zonation of canalicular bile acid concentrations. Upper panel: a central to portal gradient is established, if CMNB Fluorescein is photoactivated only in the canaliculi. Lower panel: Establishment of a portal to central gradient due to the higher excretion rate of periportal compared to pericentral hepatocytes.

This article is protected by copyright. All rights reserved 
Figure 1

A
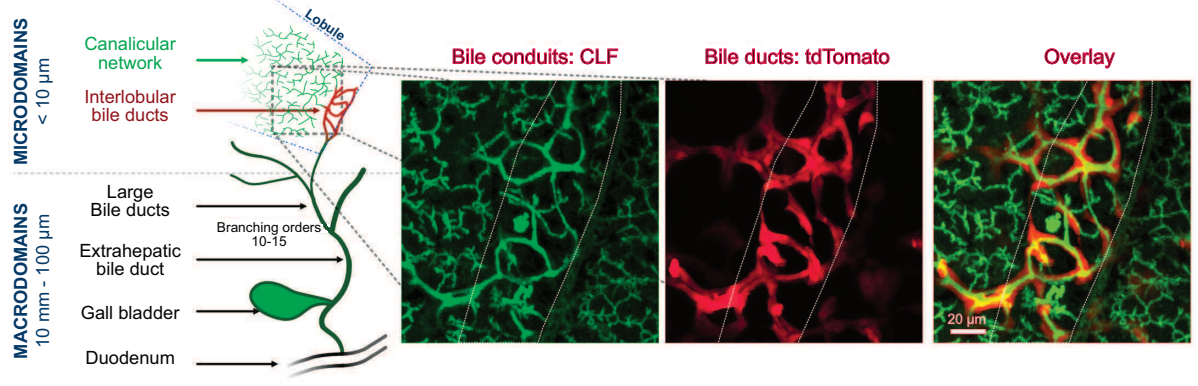

B
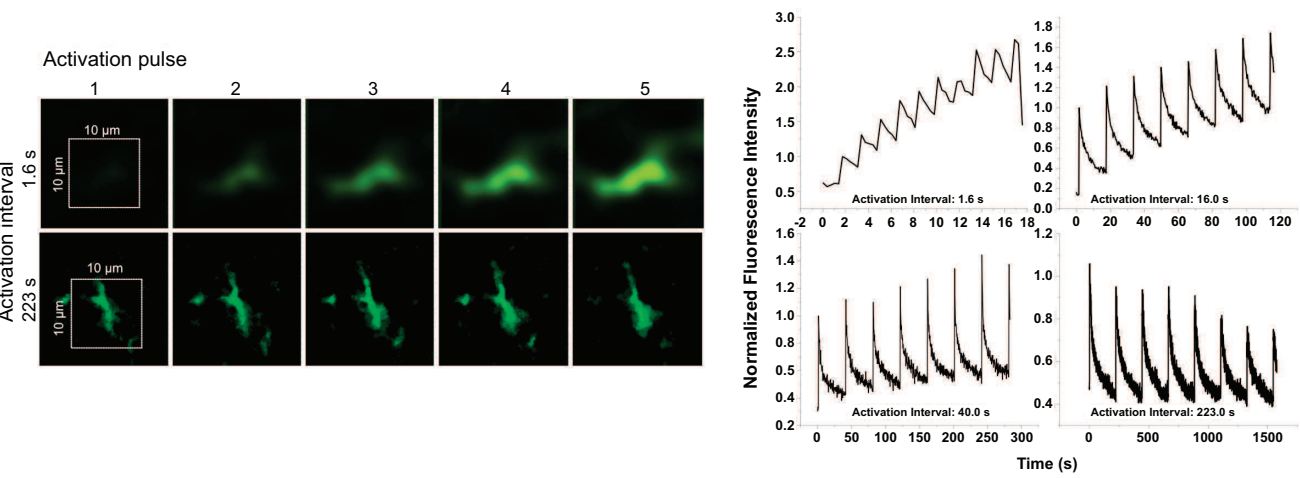

C
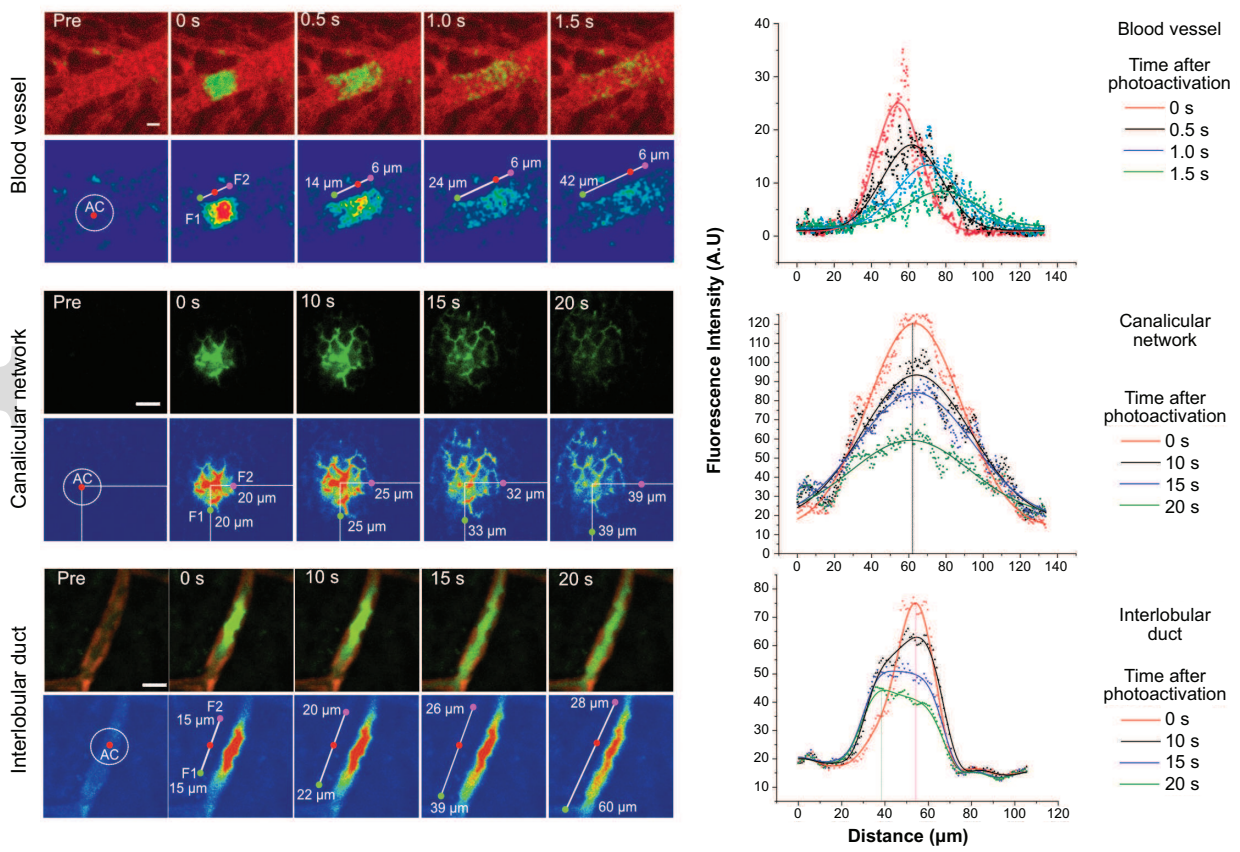

This article is protected by copyright. All rights reserved 
CMNB-Fluo
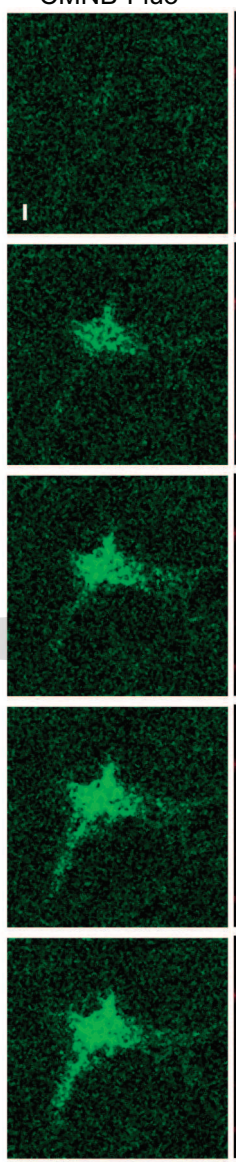

tdTomato
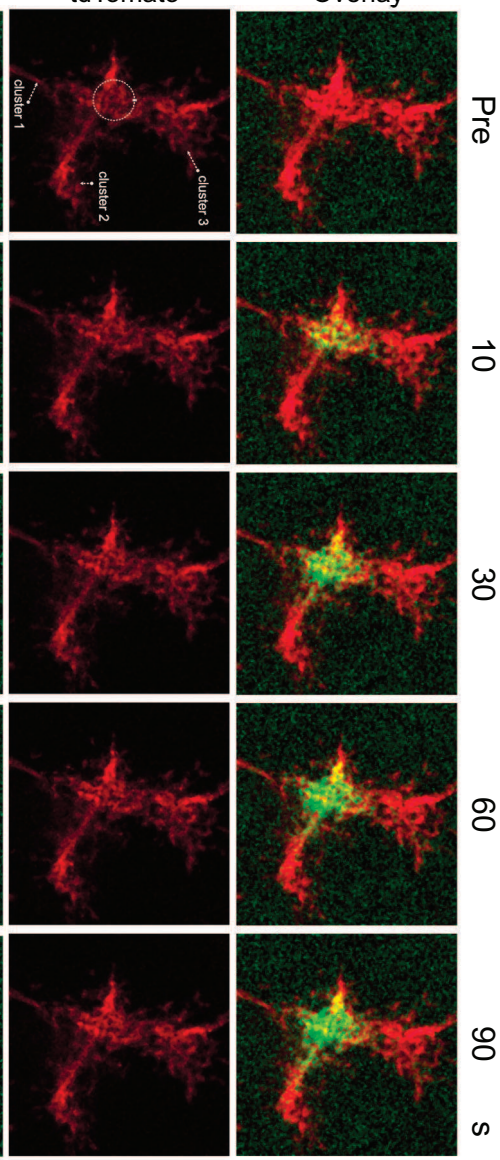

Overlay
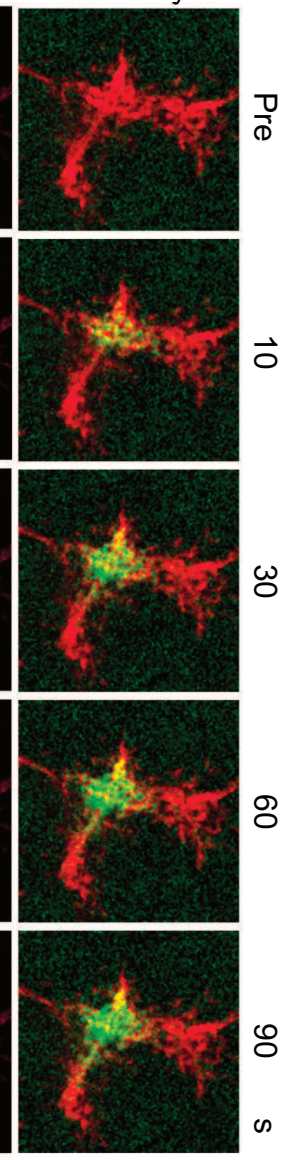

$\frac{0}{D}$

$\overrightarrow{0}$

బ.

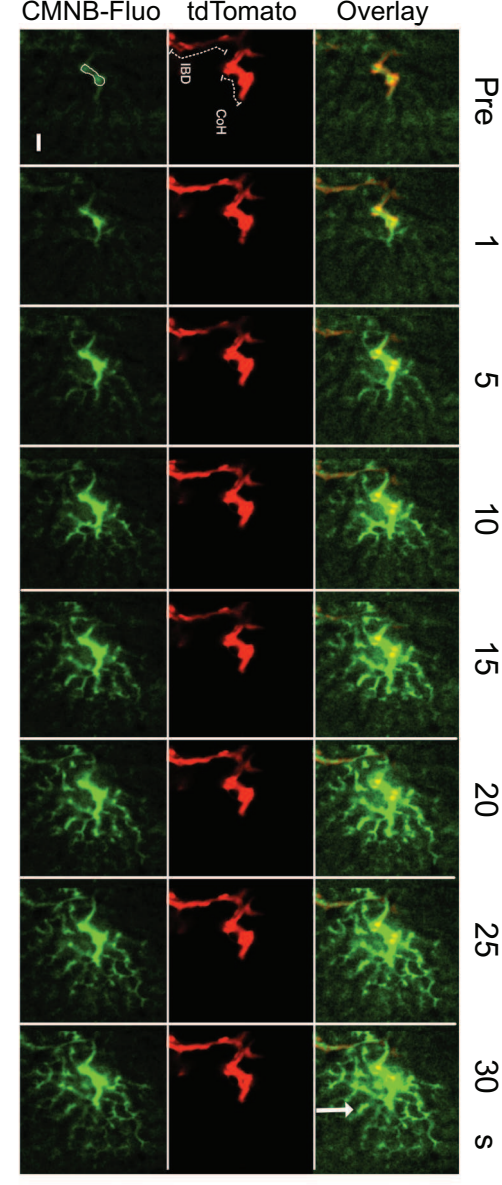

CMNB-Fluo tdTomato Overlay

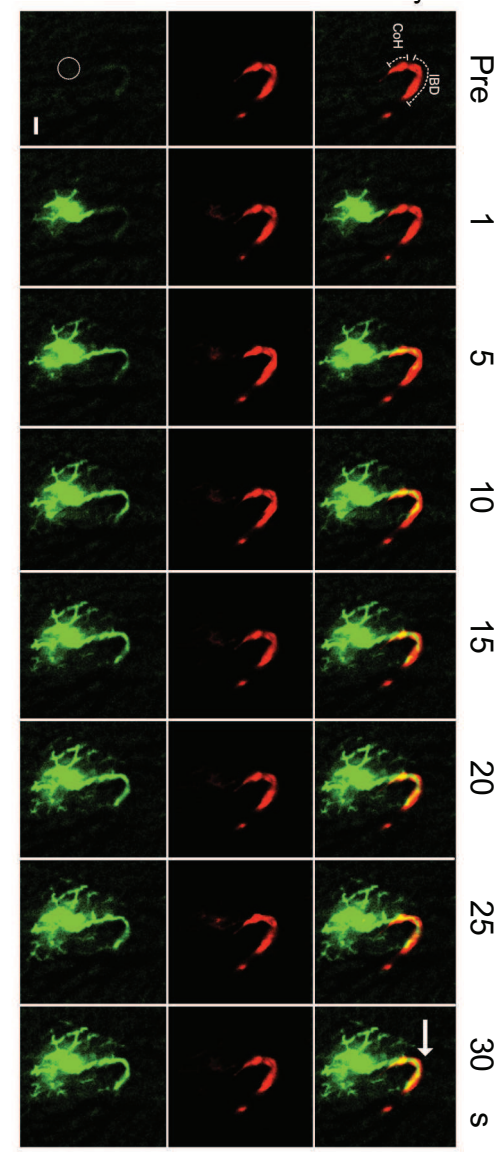


Figure 3

A

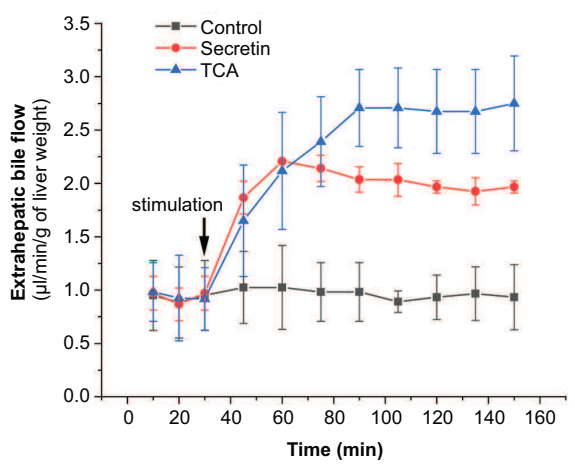

B
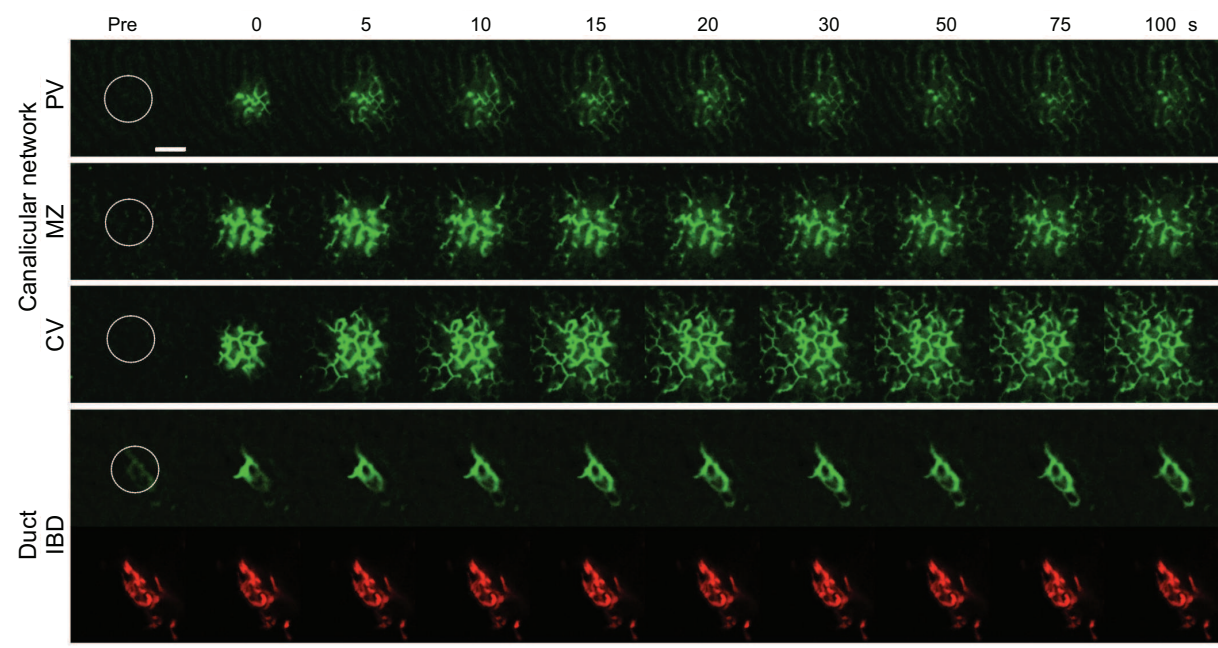

C
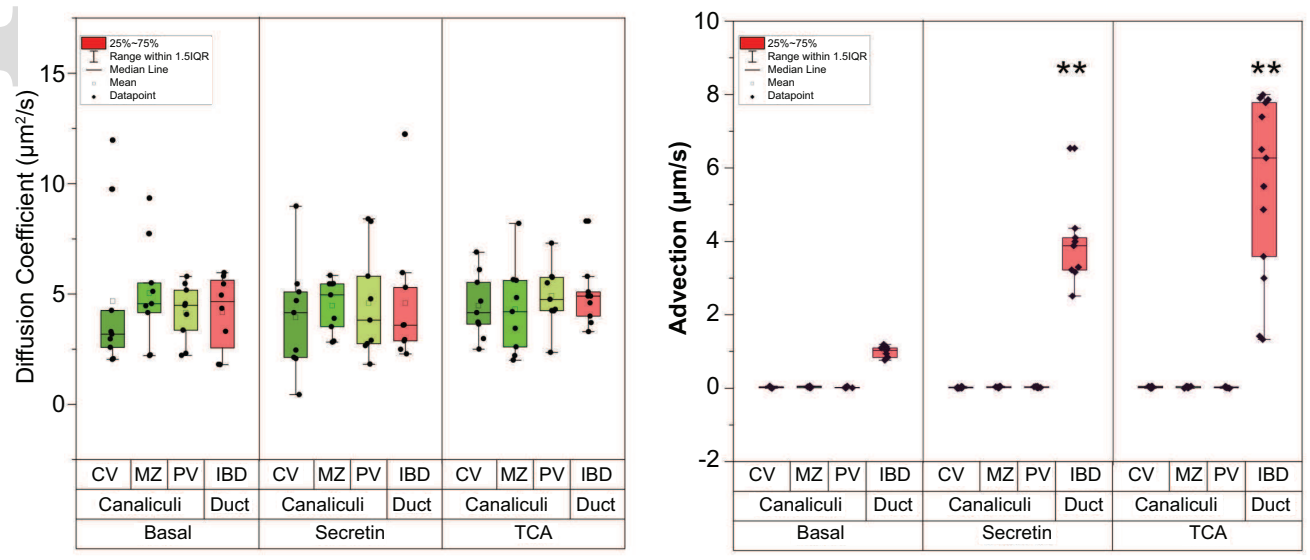
Figure 4

A
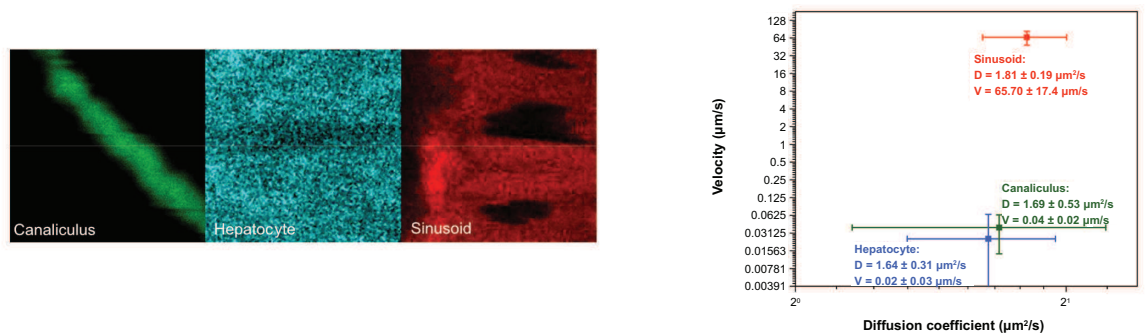

B

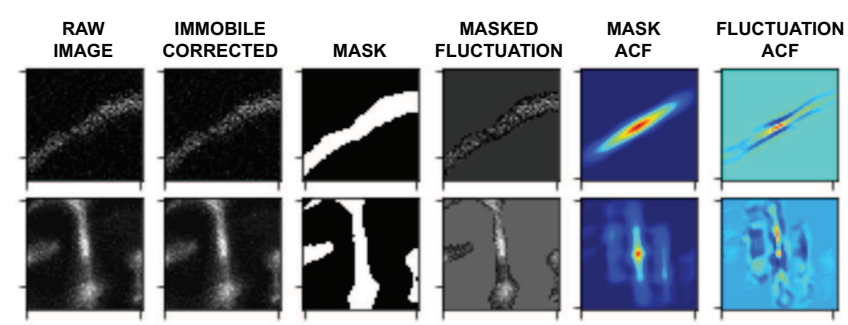

C
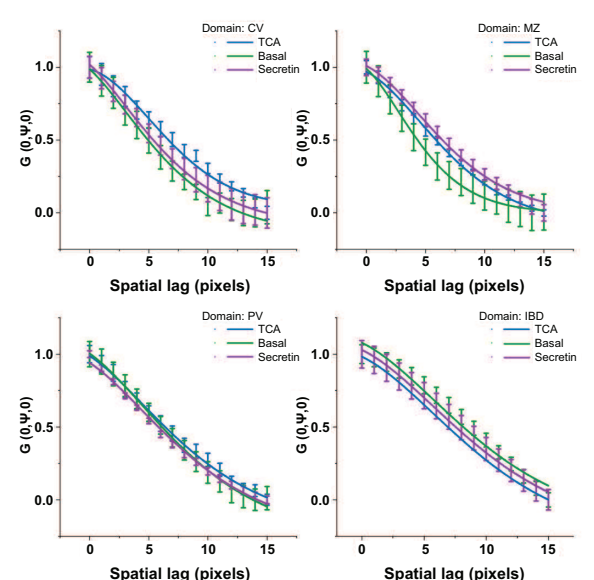

E

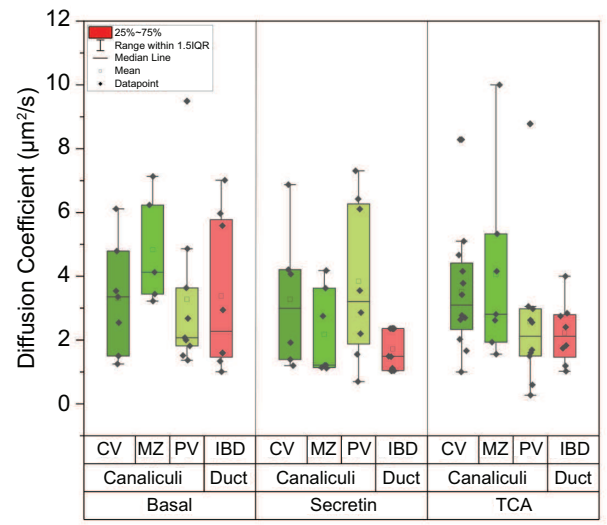

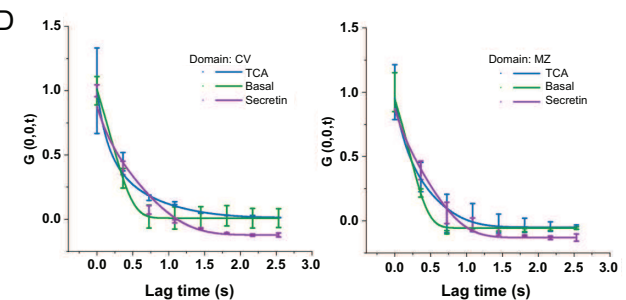

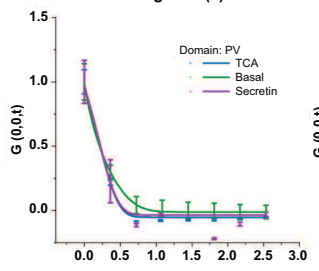

Lag time (s)
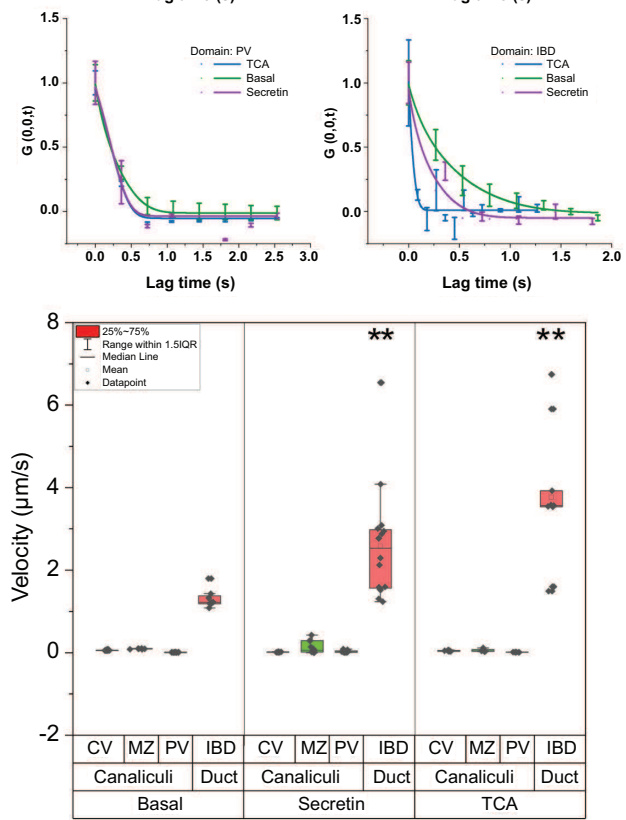
Figure 5

A

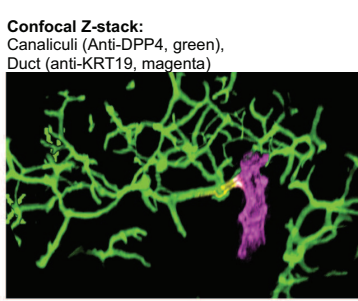

3D Wireframe representation of conduit lumin $(0.4 \mu \mathrm{m}$ mesh)
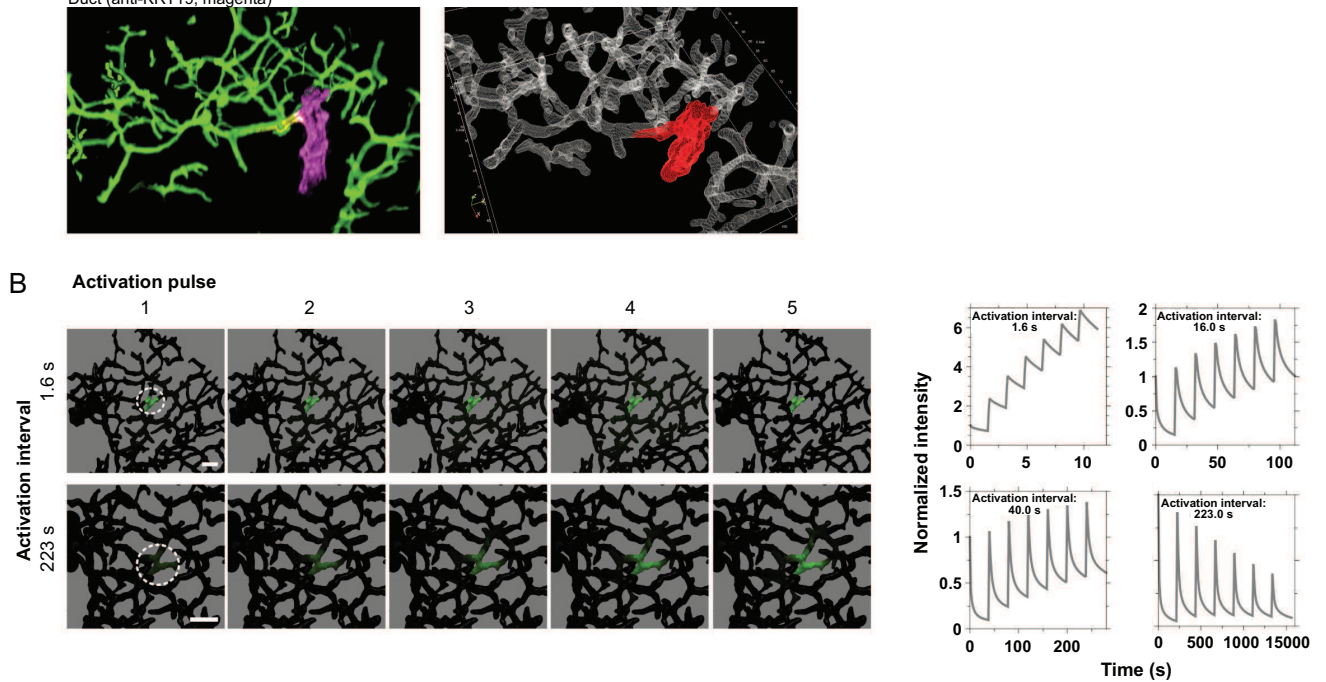

C Canalicular network Diffusion coefficient $=3 \mu \mathrm{m}^{2} / \mathrm{s}$
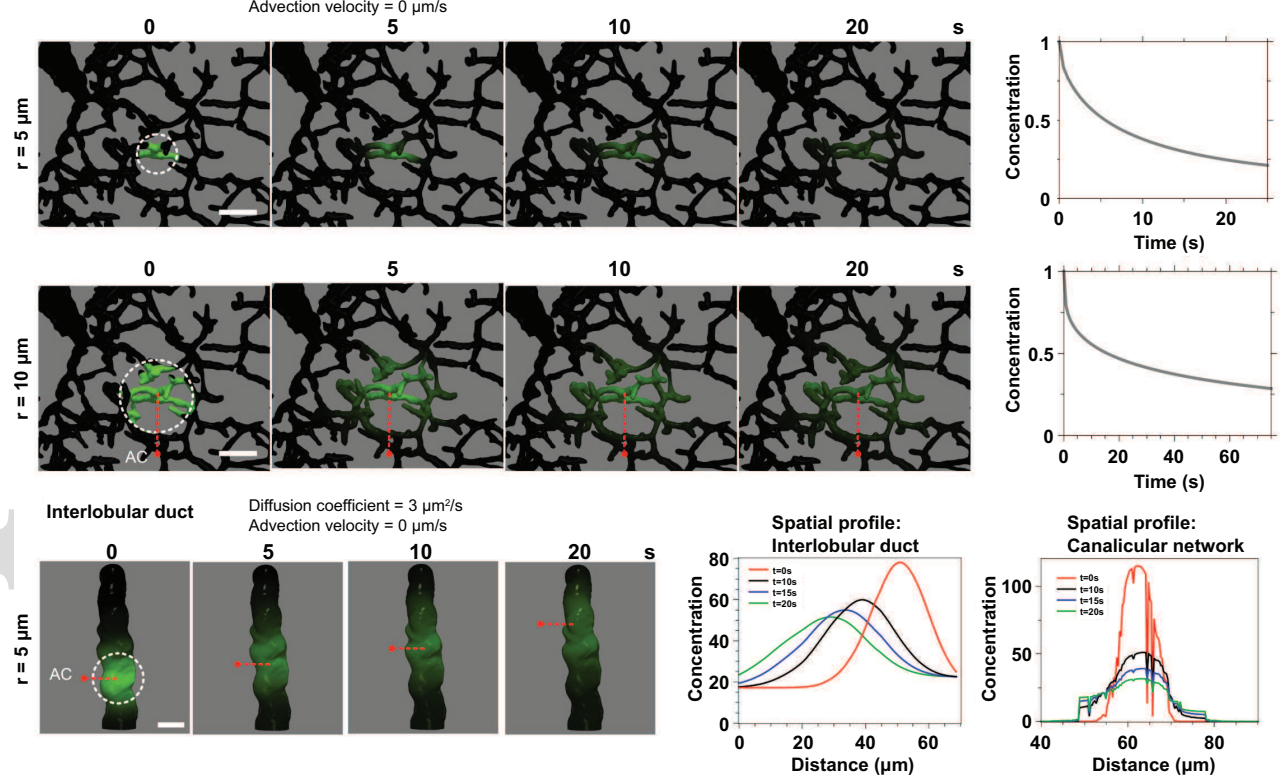

D Canal of Hering $\quad \begin{aligned} & \text { Diffusion coefficient }=3 \mu \mathrm{m}^{2} / \mathrm{s} \\ & \text { Advection velocity }=0 \mu \mathrm{m} / \mathrm{s}\end{aligned}$

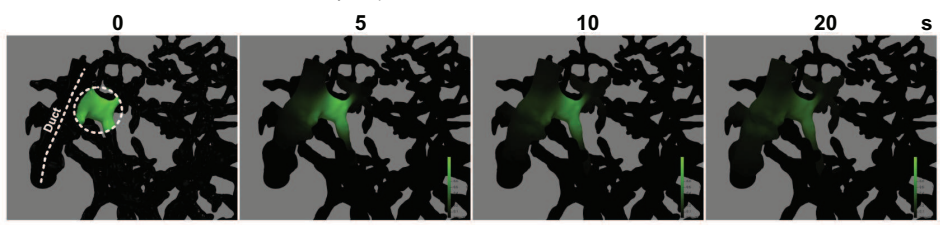


Figure 6

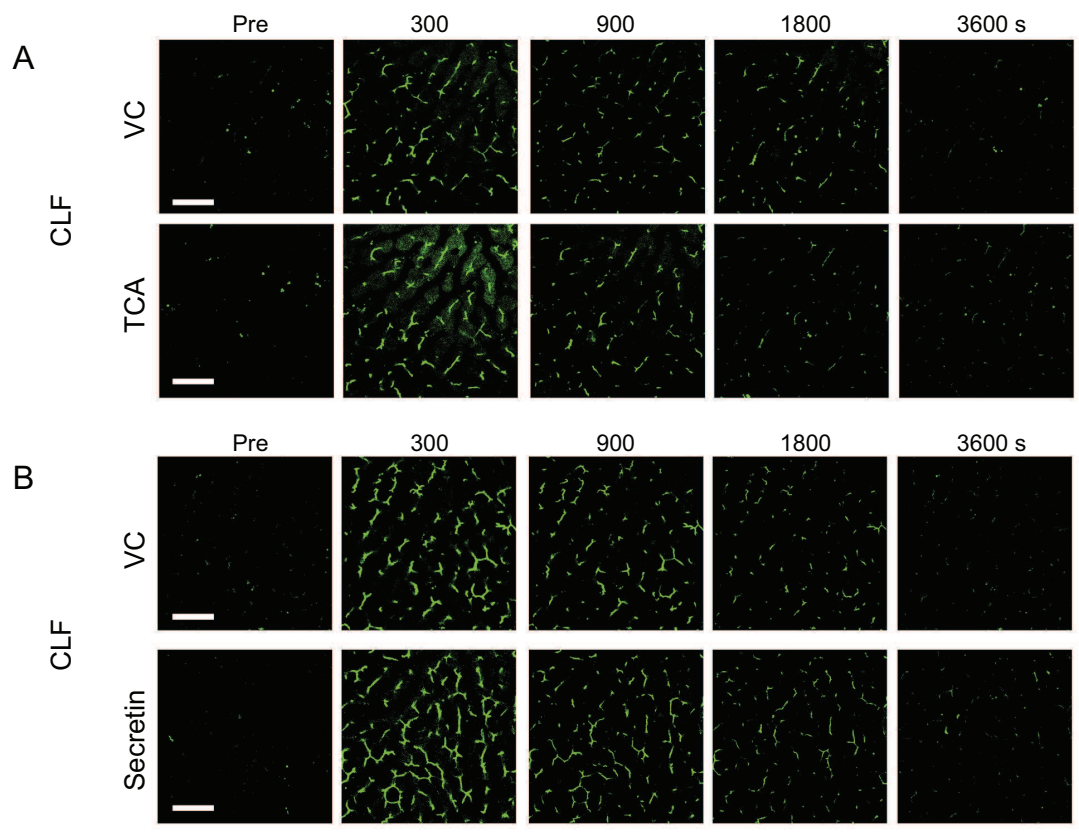

C
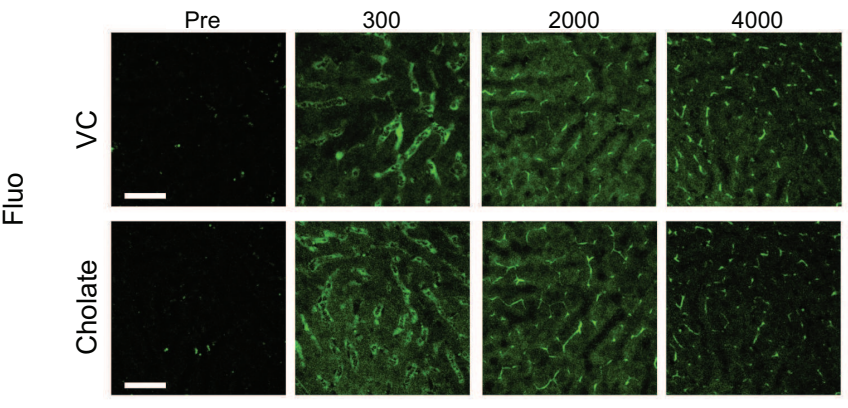

$6000 \mathrm{~s}$

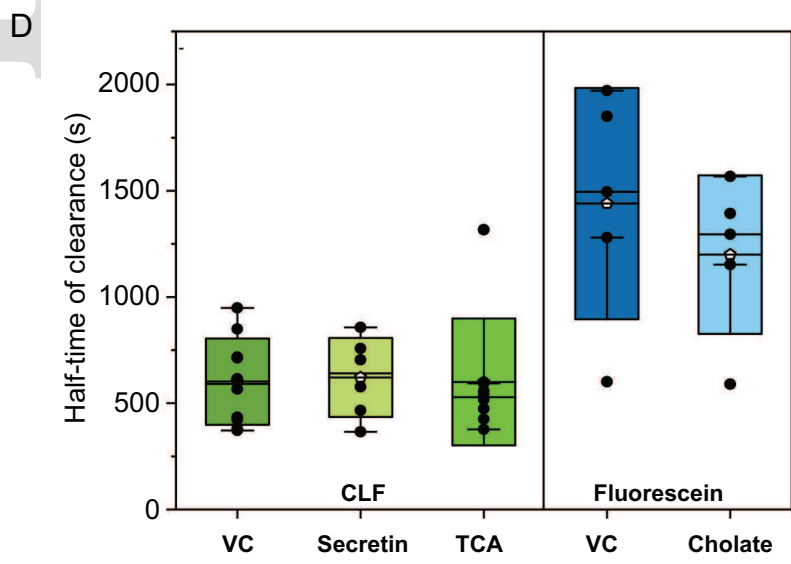

This article is protected by copyright. All rights reserved 
Figure 7
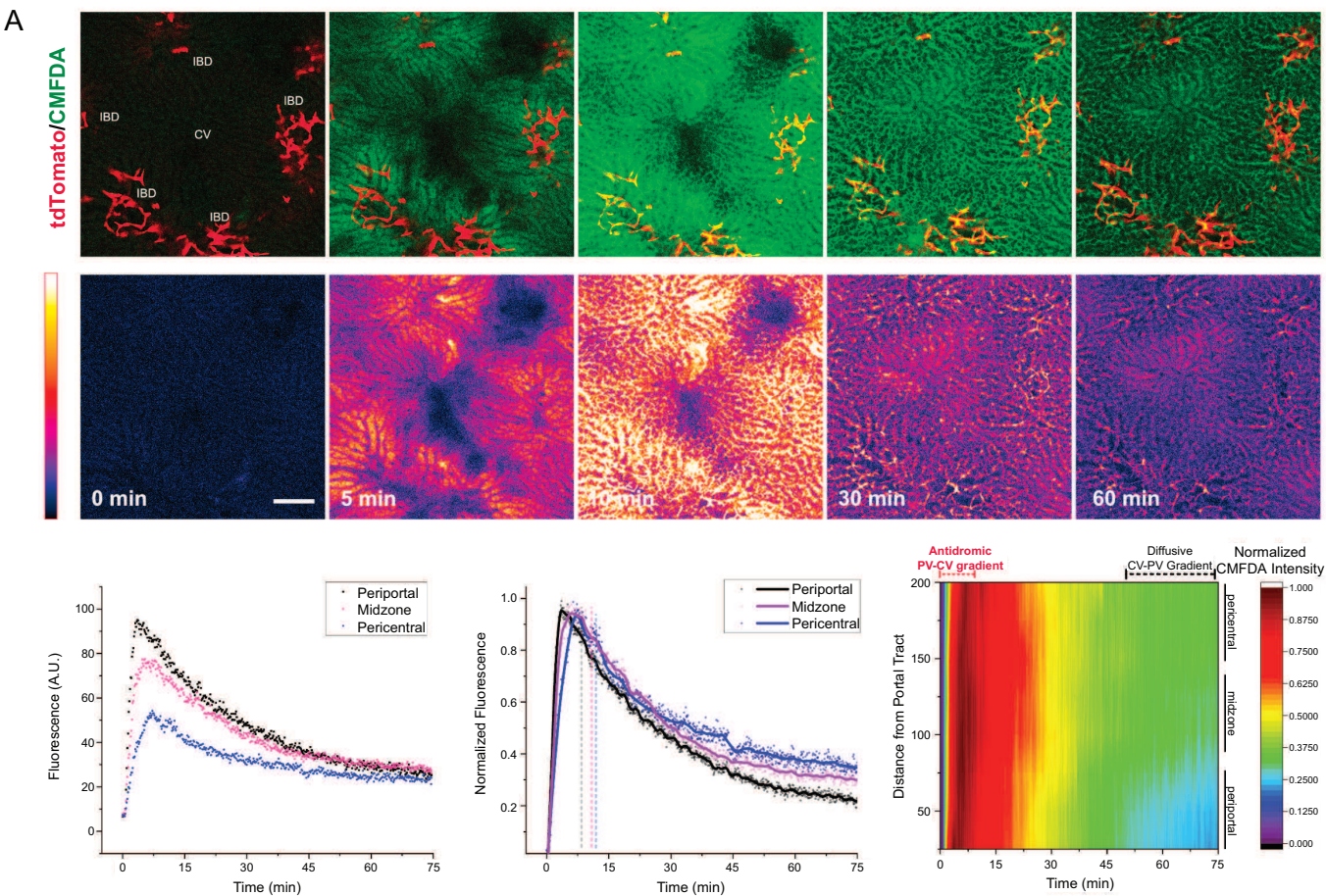

B
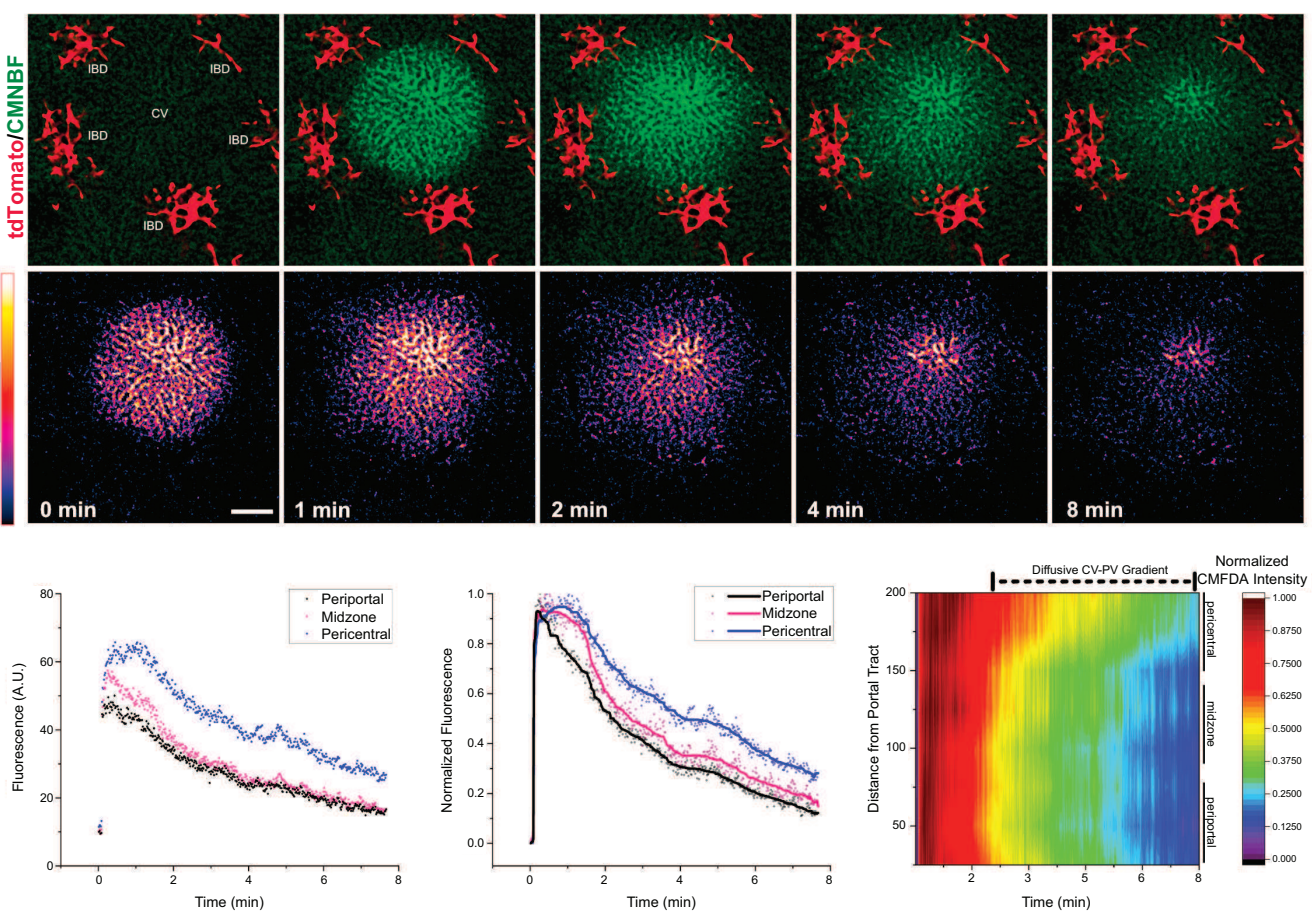

This article is protected by copyright. All rights reserved 
Figure 8

A

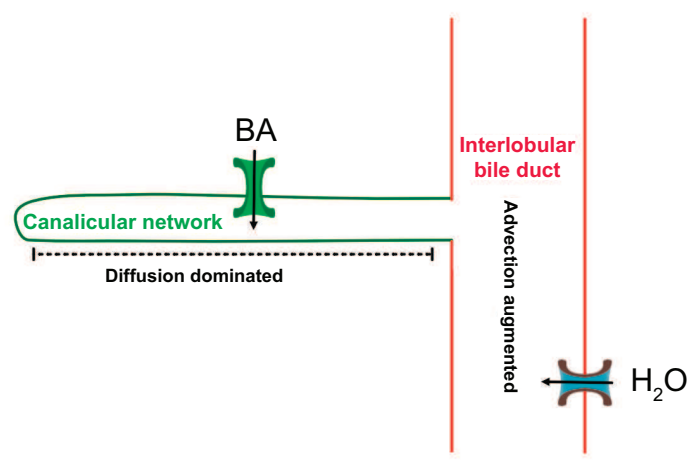

B
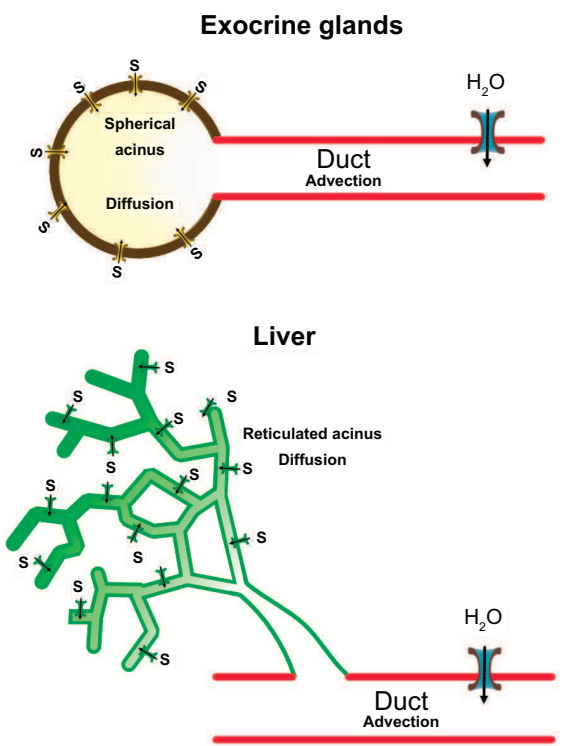

C
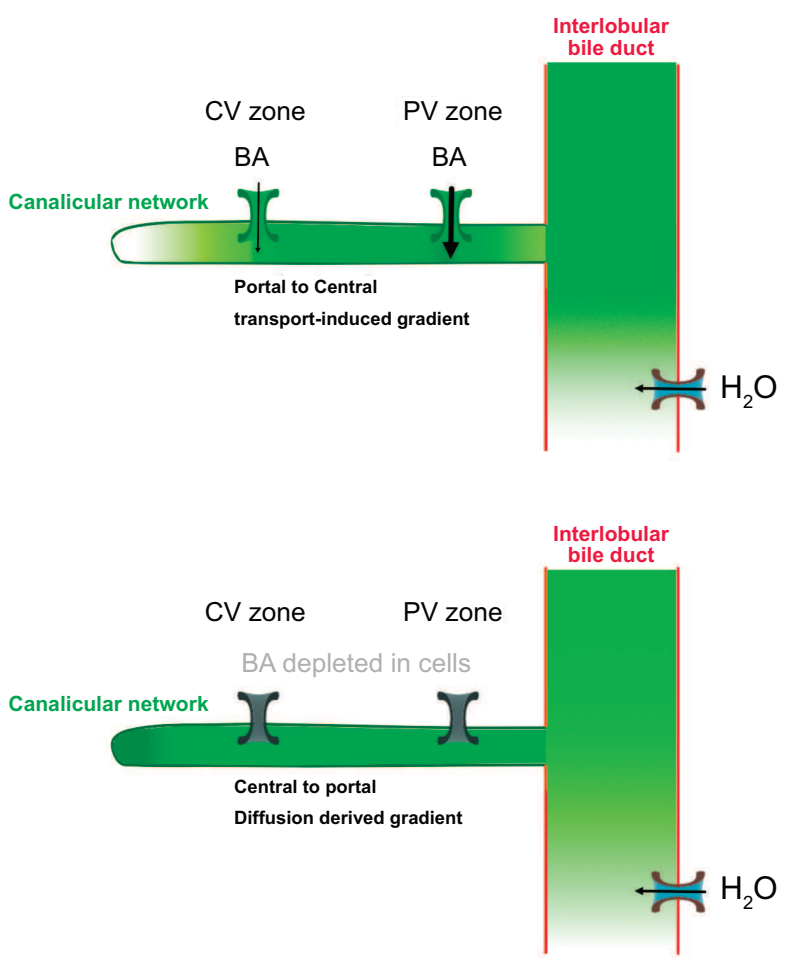

This article is protected by copyright. All rights reserved 